\title{
The functor of units of Burnside rings for $p$-groups
}

Serge Bouc

\begin{abstract}
In this paper, I describe the structure of the biset functor $B^{\times}$sending a $p$-group $P$ to the group of units of its Burnside ring $B(P)$. In particular, I show that $B^{\times}$is a rational biset functor. It follows that if $P$ is a $p$-group, the structure of $B^{\times}(P)$ can be read from a genetic basis of $P$ : the group $B^{\times}(P)$ is an elementary abelian 2-group of rank equal to the number isomorphism classes of rational irreducible representations of $P$ whose type is trivial, cyclic of order 2 , or dihedral.
\end{abstract}

Mathematics Subject Classification (2000). 19A22, 16 U60.

Keywords. Burnside ring, unit, biset functor.

\section{Introduction}

If $G$ is a finite group, denote by $B(G)$ the Burnside ring of $G$, i.e. the Grothendieck ring of the category of finite $G$-sets (see e.g. [4]). The question of the structure of the multiplicative group $B^{\times}(G)$ has been studied by T. tom Dieck ([23]), T. Matsuda ([18]), T. Matsuda and T. Miyata ([19]), T. Yoshida ([26]), by geometric and algebraic methods.

Recently, E. Yalçın wrote a very nice paper ([24]), in which he proves an induction theorem for $B^{\times}$for 2-groups, which says that if $P$ is a 2-group, then any element of $B^{\times}(P)$ is a sum of elements obtained by inflation and tensor induction from sections $(T, S)$ of $P$, such that $T / S$ is trivial or dihedral.

The main theorem of the present paper implies a more precise form of Yalçın's Theorem, but the proof is independent, and uses entirely different methods. In particular, the biset functor techniques developed in [3], [7] and [9], lead to a precise description of $B^{\times}(P)$, when $P$ is a 2-group (actually also for arbitrary $p$-groups, but the case when $p$ is odd is known to be rather trivial). The main ingredient consists of showing that $B^{\times}$is a rational biset functor, and this is done by showing that the functor $B^{\times}$(restricted to $p$-groups) is a subfunctor of the functor $\mathbb{F}_{2} R_{\mathbb{Q}}^{*}$, where $R_{\mathbb{Q}}^{*}=\operatorname{Hom}_{\mathbb{Z}}\left(R_{\mathbb{Q}}, \mathbb{Z}\right)$ denote the $\mathbb{Z}$-dual of the functor $R_{\mathbb{Q}}$ of rational representations, and $\mathbb{F}_{2} R_{\mathbb{Q}}^{*}=\mathbb{F}_{2} \otimes R_{\mathbb{Q}}^{*}$. This leads to a description of $B^{\times}(P)$ in terms of a genetic basis of $P$, or equivalently, in terms of rational irreducible representations of $P$. 
The paper is organized as follows. In Section 2, I recall the basic definitions, properties and constructions related to the Burnside ring of a finite group. Section 3 is a similar summary of the main definitions and notation on biset functors. Section 4 deals with genetic subgroups and rational biset functors. Section 5 gives a natural exposition of the biset functor structure of $B^{\times}$. In Section 6, I give cases where inflation or restriction allow for a computation of $B^{\times}(G)$ for a finite group $G$, and also some results about faithful elements in $B^{\times}(P)$ for specific $p$-groups $P$. In Section 7, I introduce a natural transformation of biset functors from $B^{\times}$to $\mathbb{F}_{2} B^{*}$. This transformation is injective, and in Section 8 , I show that the image of its restriction to the subcategory of $p$-groups is contained in the subfunctor $\mathbb{F}_{2} R_{\mathbb{Q}}^{*}$ of $\mathbb{F}_{2} B^{*}$.

This is the key result, leading to a precise description of $B^{\times}(G)$, when $G$ is a finite $p$-group, or more generally a finite nilpotent group (Theorem 8.7). The main result of Section 9 is a description of the lattice of subfunctors of the restriction of $B^{\times}$to the subcategory of $p$-groups: it is always a uniserial $p$-biset functor (even simple if $p$ is odd). This result has two interesting consequences: the first one is a complete answer to the question, raised by Yalçin ([24]), of the surjectivity of the exponential map $B(P) \rightarrow B^{\times}(P)$ for a 2-group $P$. The second one is a connection with the Dade group of $p$-groups: I show that there is a short exact sequence of $p$-biset functors involving $B^{\times}, \mathbb{F}_{2} R_{\mathbb{Q}}^{*}$ and the $\mathbb{F}_{2}$-reduction $\mathbb{F}_{2} D_{\text {tors }}^{\Omega}$ of the torsion subfunctor of the functor of relative syzygies in the Dade group.

\section{The Burnside ring of a finite group}

Details on the definitions and proofs of the results about the Burnside ring summarized in this section can be found e.g. in [4].

Definition 2.1. Let $G$ be a finite group. The Burnside group $B(G)$ is the quotient of the free abelian group on the set of isomorphism classes of finite $G$-sets, by the subgroup generated by the elements $[X \sqcup Y]-[X]-[Y]$, where $X$ and $Y$ are $G$-sets and $X \sqcup Y$ is their disjoint union, and where $[X]$ denotes the isomorphism class of the $G$-set $X$.

The product of the $G$-sets $X$ and $Y$ is the cartesian product $X \times Y$, for diagonal $G$-action. This construction extends to a product on $B(G)$, giving it the structure of a commutative ring, called the Burnside ring of $G$. The identity element of $B(G)$ is the class of a $G$-set of cardinality 1 . It will be denoted by $1_{B}$.

2.2. The canonical basis. The elements of $B(G)$ can be written as differences [X][Y] for suitable finite $G$-sets $X$ and $Y$. One can show that the abelian group $B(G)$ is a free abelian group on the set of isomorphism classes of transitive $G$-sets. Such a transitive $G$-set is isomorphic to the set of cosets $G / H$, for some subgroup $H$ of $G$. 
If $H$ and $K$ are subgroups of $G$, then the $G$-sets $G / H$ and $G / K$ are isomorphic if and only if the subgroups $H$ and $K$ are conjugate in $G$. Thus:

Definition 2.3. Let $\left[s_{G}\right]$ be a set of representatives of conjugacy classes of subgroups of $G$. Then the elements $[G / H]$, for $H \in\left[s_{G}\right]$, form a $\mathbb{Z}$-basis of $B(G)$, called the canonical basis of $B(G)$.

Note in particular that $1_{B}=[G / G]$.

If $H$ is a subgroup of $G$, then the correspondence mapping [X] to the cardinality $\left|X^{H}\right|$ of the set $X^{H}$ of elements of $X$ which are fixed by $H$ can be extended uniquely to an additive map $\phi_{H}: B(G) \rightarrow \mathbb{Z}$. The map $\phi_{H}$ is actually a morphism of rings with identity elements. Conversely, any ring homomorphism from $B(G)$ to $\mathbb{Z}$ is equal to $\phi_{H}$, for some subgroup $H$ of $G$.

Definition 2.4. The product ring $C(G)=\prod_{H \in\left[s_{G}\right]} \mathbb{Z}$ is called the ghost ring of $G$. The product map $\phi=\prod_{H \in\left[s_{G}\right]} \phi_{H}$ is called the ghost map.

Theorem 2.5 (Burnside). The ghost map is injective.

This theorem means that an element $a$ of $B(G)$ is characterized by the integers $\left|a^{H}\right|$, for all subgroups $H$ of $G$. Moreover, since $B(G)$ and $C(G)$ are free abelian groups with the same rank, the cokernel of the ghost map is finite. A theorem of Dress ([15]) characterizes the image of $B(G)$ inside $C(G)$.

2.6. Idempotents. Another consequence of Theorem 2.5 is that the ghost map $\phi$ becomes an isomorphism after extension of scalars to $\mathbb{Q}$ : in other words, the map

$$
\mathbb{Q} \phi=\prod_{H \in\left[s_{G}\right]} \mathbb{Q} \phi_{H}: \mathbb{Q} B(G)=\mathbb{Q} \otimes_{\mathbb{Z}} B(G) \rightarrow \mathbb{Q} C(G)=\mathbb{Q} \otimes_{\mathbb{Z}} C(G) \cong \prod_{H \in\left[s_{G}\right]} \mathbb{Q}
$$

is a $\mathbb{Q}$-algebra isomorphism. In particular $\mathbb{Q} B(G)$ is a split semi-simple commutative $\mathbb{Q}$-algebra. Its primitive idempotents are indexed by the set $\left[s_{G}\right]$. The idempotent $e_{H}^{G}$ indexed by the subgroup $H$ is characterized by the fact that for any $a \in \mathbb{Q} B(G)$, the product $a e_{H}^{G}$ is equal to $\left|a^{H}\right| e_{H}^{G}$, where $\left|a^{H}\right|=\left(\mathbb{Q} \phi_{H}\right)(a)$.

It has been shown by Gluck ([16]) and independently by Yoshida ([25]) that $e_{H}^{G}$ can be expressed as

$$
e_{H}^{G}=\frac{1}{\left|N_{G}(H)\right|} \sum_{K \subseteq H}|K| \mu(K, H)[G / K],
$$

where $\mu(K, H)$ is the value of the Möbius function of the poset of all subgroups of $G$, ordered by inclusion of subgroups. 
2.7. Prime spectrum. The prime spectrum of $B(G)$, and more generally of localizations of $B(G)$ with respect to any set of prime numbers, have been described by Dress ([15]). In particular, Dress showed that the primes spectrum of $B(G)$ is connected if and only if the group $G$ is solvable. Equivalently, the identity element $1_{B}=[G / G]$ is a primitive idempotent of $B(G)$ if and only if $G$ is solvable.

Notation 2.8. If $G$ is a finite group, then let $B^{\times}(G)$ denote the group of units of $B(G)$.

It follows from Theorem 2.5 that an element $u$ of $B(G)$ is in $B^{\times}(G)$ if and only if $\left|u^{H}\right|= \pm 1$ for any subgroup $H$ of $G$, or equivalently, if $u^{2}=1_{B}$. In particular, the group $B^{\times}(G)$ of units of the ring $B(G)$ is always an elementary abelian 2-group.

It also follows that the mappings $u \mapsto\left(1_{B}-u\right) / 2$ and $e \mapsto 1_{B}-2 e$ are mutual inverse bijections between $B^{\times}(G)$ and the set of idempotents $e$ of $\mathbb{Q} B(G)$ such that $2 e \in B(G)$.

The determination of $B^{\times}(G)$ for an arbitrary finite group $G$ is an open problem in general. The following argument, due to tom Dieck ([23], Proposition 1.5.1) illustrates its difficulty: suppose that $G$ has odd order. Now if $u \in B^{\times}(G)$, the idempotent $e=\left(1_{B}-u\right) / 2$ of $\mathbb{Q} B(G)$ is such that $2 e \in B(G)$. But $|G| e$ is also in $B(G)$, by the above formulae for primitive idempotents in $\mathbb{Q} B(G)$. Since $|G|$ is odd, it follows that $e \in B(G)$.

Conversely, if $e$ is an idempotent in $B(G)$, then $1_{B}-2 e \in B^{\times}(G)$. In other words, when $G$ is odd, there is a one to one correspondence between $B^{\times}(G)$ and the idempotents of $B(G)$. So proving that $G$ is solvable is equivalent to proving that $B^{\times}(G)=\left\{ \pm 1_{B}\right\}$, by the above theorem of Dress. Thus:

Theorem 2.9 (tom Dieck). Feit-Thompson's theorem is equivalent to the statement that if $G$ has odd order, then $B^{\times}(G)=\left\{ \pm 1_{B}\right\}$.

So even the question of knowing when $B^{\times}(G)=\left\{ \pm 1_{B}\right\}$ is highly non-trivial. Note however that for an odd order $p$-group, this becomes rather obvious (see Lemma 6.3).

There are not so many general results on the structure of $B^{\times}(G)$ : Yoshida ([26]) stated some reduction theorems, using 2-perfect subgroups of $G$. He also gave the following nice characterization of the image of $B^{\times}(G)$ inside $C^{\times}(G)$ :

Theorem 2.10 (Yoshida). Let $G$ be a finite group. Then the element $u$ of $C^{\times}(G)=$ $\prod_{H \in\left[s_{G}\right\}}\{ \pm 1\}$ lies in $\phi\left(B^{\times}(G)\right)$ if and only if, for each subgroup $H$ of $G$, the mapping $x H \in N_{G}(H) / H \mapsto \frac{u_{\langle x\rangle}}{u_{H}} \in\{ \pm 1\}$ is a group homomorphism.

For abelian groups, Matsuda ([18]) proved the following: 
Theorem 2.11 (Matsuda). Let $G$ be an abelian group. Then $B^{\times}(G)$ is elementary abelian of order $2^{1+n}$, where $n$ is the number of subgroups of index 2 of $G$.

In the same article, Matsuda computes $B^{\times}(G)$ in some particular cases, e.g. when $G$ is dihedral, or isomorphic to the product of two dihedral groups (with some restrictions on the orders). Matsuda and Miyata ([19]) have shown that if $G$ is the semi-direct product of a normal subgroup $H$ by an odd order group $K$, then $B^{\times}(G)$ is isomorphic to the subgroup $B^{\times}(H)^{K}$ of elements of $B^{\times}(H)$ which are fixed by the natural action of $K$. Proposition 6.5 will generalize this result to the case of a normal subgroup $H$ of odd index in $G$.

2.12. Restriction, inflation, fixed points, tensor induction. The main tool in the study of the group of units of the Burnside ring is the existence natural group homomorphisms $B^{\times}\left(G_{1}\right) \rightarrow B^{\times}\left(G_{2}\right)$ for different groups $G_{1}$ and $G_{2}$. These maps correspond to various situations:

- If $H$ is a subgroup of $G$, then the restriction to $H$ of the $G$-action induces a linear map $\operatorname{Res}_{H}^{G}: B(G) \rightarrow B(H)$, which is actually a ring homomorphism, preserving identity elements. It follows that it maps units to units, and this gives a restriction map also denoted by $\operatorname{Res}_{H}^{G}$ from $B^{\times}(G)$ to $B^{\times}(H)$.

- If $N$ is a normal subgroup of $G$, then the inflation of $(G / N)$-sets to $G$-sets with a trivial $N$-action induces a map $\operatorname{Inf}_{G / N}^{G}: B(G / N) \rightarrow B(G)$, which is a ring homomorphism, and preserves identity elements. This gives an inflation map $\operatorname{Inf}_{G / N}^{G}: B^{\times}(G / N) \rightarrow B^{\times}(G)$.

- If $N$ is a normal subgroup of $G$, and if $X$ is a $G$-set, then the set $X^{N}$ of elements of $X$ which are fixed by $N$ is a $(G / N)$-set. This gives a map $B(G) \rightarrow B(G / N)$, called the fixed points map, or the deflation map. This is again a ring homomorphism, and it preserves identity elements. So it induces a deflation map $\operatorname{Def}_{G / N}^{G}: B^{\times}(G) \rightarrow$ $B^{\times}(G / N)$.

- The three previous operations $B^{\times}\left(G_{1}\right) \rightarrow B^{\times}\left(G_{2}\right)$ are the restrictions to units groups of a ring homomorphism $B\left(G_{1}\right) \rightarrow B\left(G_{2}\right)$. This is no longer the case for the fourth operation, which is an induction operation: if $H$ is a subgroup of $G$, and if $Y$ is an $H$-set, then the set $\operatorname{Hom}_{H}(G, Y)$ of $H$-equivariant maps from $G$ to $Y$ can be endowed with the $G$-action defined by $(g f)(x)=f(x g)$, for $g, x \in G$ and $f \in \operatorname{Hom}_{H}(G, Y)$. This construction can be extended to give a map $\operatorname{Ten}_{H}^{G}: B(H) \rightarrow$ $B(G)$, called tensor induction, which is not additive in general, but still multiplicative, and preserves identity elements. This extension to a multiplicative map between Burnside rings can be achieved by different means. One of them will be detailed in Section 5. By restriction to the corresponding groups of units, the map $\operatorname{Ten}_{H}^{G}$ gives a map $B^{\times}(H) \rightarrow B^{\times}(G)$, called the tensor induction map, or the multiplicative induction. It has often been denoted by $\operatorname{Jnd}_{H}^{G}$. It will be denoted by $\operatorname{Ten}_{H}^{G}$ in this paper. 
- Together with the obvious map Iso $(\varphi): B^{\times}\left(G_{1}\right) \rightarrow B^{\times}\left(G_{2}\right)$ associated to a group isomorphism $\varphi: G_{1} \rightarrow G_{2}$, the above four kind of maps can be given a unified definition, using bisets. This leads to the structure of biset functor on $B^{\times}$, and will be detailed in Section 5 .

2.13. Yalçın's theorem. The present paper originates in a recent article by Yalçın ([24]), as already mentioned in the introduction. The main result of Yalçın's paper is an induction theorem, stated below with the following notation: if $(H, K)$ is a section of the group $G$, i.e. if $H$ is a subgroup of $G$ and $K$ is a normal subgroup of $H$ (see Definition 3.4), denote by Teninf ${ }_{H / K}^{G}$ the map from $B^{\times}(H / K)$ to $B^{\times}(G)$ obtained by composing the inflation map $\operatorname{Inf}_{H / K}^{H}$ with the tensor induction map $\operatorname{Ten}_{H}^{G}$. Then:

Theorem 2.14 (Yalçın[24]). Let $G$ be a finite 2-group, and let $\mathscr{H}$ denote the set of sections $(H, K)$ of $G$ such that $H=K$ or $H / K$ is a dihedral group of order at least 16. Then the map

$$
\bigoplus_{(H, K) \in \mathcal{H}} \operatorname{Teninf}_{H / K}^{G}: \bigoplus_{(H, K) \in \mathcal{H}} B^{\times}(H / K) \rightarrow B^{\times}(G)
$$

is surjective.

The present paper can be viewed as a refinement of this theorem, by a systematic use of the formalism of biset functors.

Notation 2.15. In the remainder of this paper:

- The brackets will be omitted in the Burnside ring. So there will be no notational difference between a finite $G$-set $X$ and its isomorphism class.

- If $a \in B(G)$, then the image of $a$ under the above map $\phi_{H}$ will be denoted by $\left|a^{H}\right|$. This can be any element of $\mathbb{Z}$.

- The group $B(G)$ will be identified with its image $1 \otimes_{\mathbb{Z}} B(G)$ inside $\mathbb{Q} B(G)=$ $\mathbb{Q} \otimes_{\mathbb{Z}} B(G)$.

\section{Biset functors}

Notation and Definition 3.1. Denote by $\mathcal{C}$ the following category:

- The objects of $\mathcal{C}$ are the finite groups.

- If $G$ and $H$ are finite $p$-groups, then $\operatorname{Hom}_{\mathcal{C}}(G, H)=B\left(H \times G^{\mathrm{op}}\right)$ is the Burnside group of finite $(H, G)$-bisets. An element of this group is called a virtual $(H, G)$-biset. 
- The composition of morphisms is $\mathbb{Z}$-bilinear, and if $G, H, K$ are finite groups, if $U$ is a finite $(H, G)$-biset, and $V$ is a finite $(K, H)$-biset, then the composition of (the isomorphism classes of) $V$ and $U$ is the (isomorphism class) of $V \times{ }_{H} U$. The identity morphism $\operatorname{Id}_{G}$ of the group $G$ is the class of the set $G$, with left and right action by multiplication.

If $p$ is a prime number, denote by $\mathcal{C}_{p}$ the full subcategory of $\mathcal{C}$ whose objects are finite $p$-groups.

Let $\mathcal{F}$ denote the category of additive functors from $\mathcal{C}$ to the category $\mathbb{Z}$-Mod of abelian groups. An object of $\mathcal{F}$ is called a biset functor. Similarly, denote by $\mathcal{F}_{p}$ the category of additive functors from $\mathcal{C}_{p}$ to $\mathbb{Z}$-Mod. An object of $\mathcal{F}_{p}$ will be called a p-biset functor.

If $F$ is an object of $\mathcal{F}$, if $G$ and $H$ are finite groups, and if $\varphi \in \operatorname{Hom}_{\mathcal{C}}(G, H)$, then the image of $w \in F(G)$ by the map $F(\varphi)$ will generally be denoted by $\varphi(w)$. The composition $\psi \circ \varphi$ of morphisms $\varphi \in \operatorname{Hom}_{\mathcal{C}}(G, H)$ and $\psi \in \operatorname{Hom}_{\mathcal{C}}(H, K)$ will also be denoted by $\psi \times_{H} \varphi$.

Notation 3.2. Throughout this paper, the symbol 1 denotes a trivial group. The Burnside biset functor will be denoted by $B$. The functor of rational representations (see Section 1 of [7]) will be denoted by $R_{\mathbb{Q}}$. The restriction of $B$ and $R_{\mathbb{Q}}$ to $\mathcal{C}_{p}$ will also be denoted by $B$ and $R_{\mathbb{Q}}$.

Recall that the Burnside biset functor $B$ is defined as follows: its value at the finite group $G$ is equal to the Burnside group $B(G)$. If $H$ and $G$ are finite groups, if $U$ is a finite $(H, G)$-biset, and if $X$ is a finite $G$-set, then $U \times_{G} X$ is a finite $H$-set, and this correspondence induces a linear map $B(U): B(G) \rightarrow B(H)$. This construction can be extended to give a map $B(\varphi): B(G) \rightarrow B(H)$ associated to any $\varphi \in B\left(H \times G^{\mathrm{op}}\right)$. The Burnside functor $B$ is isomorphic to the Yoneda biset functor $\operatorname{Hom}_{\mathcal{C}}(\mathbf{1},-)$.

3.3. Examples. Recall that this formalism of bisets gives a single framework for the usual operations of induction, restriction, inflation, deflation, and transport by isomorphism via the following correspondences:

- If $H$ is a subgroup of $G$, then let $\operatorname{Ind}_{H}^{G} \in \operatorname{Hom}_{\mathcal{C}}(H, G)$ denote the set $G$, with left action of $G$ and right action of $H$ by multiplication.

- If $H$ is a subgroup of $G$, then let $\operatorname{Res}_{H}^{G} \in \operatorname{Hom}_{\mathcal{C}}(G, H)$ denote the set $G$, with left action of $H$ and right action of $G$ by multiplication.

- If $N \unlhd G$, and $H=G / N$, then let $\operatorname{Inf}_{H}^{G} \in \operatorname{Hom}_{\mathcal{C}}(H, G)$ denote the set $H$, with left action of $G$ by projection and multiplication, and right action of $H$ by multiplication. 
- If $N \unlhd G$, and $H=G / N$, then let $\operatorname{Def}_{H}^{G} \in \operatorname{Hom}_{\mathcal{C}}(G, H)$ denote the set $H$, with left action of $H$ by multiplication, and right action of $G$ by projection and multiplication.

- If $\varphi: G \rightarrow H$ is a group isomorphism, then $\operatorname{let}_{\operatorname{Iso}_{G}^{H}}^{H}=\operatorname{Iso}_{G}^{H}(\varphi) \in \operatorname{Hom}_{\mathcal{C}}(G, H)$ denote the set $H$, with left action of $H$ by multiplication, and right action of $G$ by taking image by $\varphi$, and then multiplying in $H$.

Definition 3.4. A section of the group $G$ is a pair $(T, S)$ of subgroups of $G$ such that $S \unlhd T$.

Notation 3.5. If $(T, S)$ is a section of $G$, set

$$
\operatorname{Indinf}_{T / S}^{G}=\operatorname{Ind}_{T}^{G} \operatorname{Inf}_{T / S}^{T} \text { and } \operatorname{Defres}_{T / S}^{G}=\operatorname{Def}_{T / S}^{T} \operatorname{Res}_{T}^{G} .
$$
biset.

Then $\operatorname{Indinf}_{T / S}^{G} \cong G / S$ as $(G, T / S)$-biset, and Defres ${ }_{T / S}^{G} \cong S \backslash G$ as $(T / S, G)$ -

Notation 3.6. Let $G$ and $H$ be groups, let $U$ be an $(H, G)$-biset, and let $u \in U$. If $T$ is a subgroup of $H$, set

$$
T^{u}=\{g \in G \mid \text { there exists } t \in T \text { with } t u=u g\} .
$$

This is a subgroup of $G$. Similarly, if $S$ is a subgroup of $G$, set

$$
{ }^{u} S=\{h \in H \mid \text { there exists } s \in S \text { with } u s=h u\} .
$$

This is a subgroup of $H$.

Lemma 3.7. Let $G$ and $H$ be groups, let $U$ be an $(H, G)$-biset, and let $S$ be a subgroup of $G$. Then there is an isomorphism of $\mathrm{H}$-sets

$$
U / S=\bigsqcup_{u \in[H \backslash U / S]} H /{ }^{u} S,
$$

where $[H \backslash U / S]$ is a set of representatives of $(H, S)$-orbits on $U$.

Proof. Indeed $H \backslash U / S$ is the set of orbits of $H$ on $U / S$, and ${ }^{u} S$ is the stabilizer of $u S$ in $H$.

3.8. Opposite bisets. If $G$ and $H$ are finite groups, and if $U$ is a finite $(H, G)$-biset, then let $U^{\mathrm{op}}$ denote the opposite biset; as a set, it is equal to $U$, and it is a $(G, H)$-biset for the following action:

$$
\text { g.u.h (in } \left.U^{\mathrm{op}}\right)=h^{-1} u g^{-1} \text { (in } U \text { ) for all } h \in H, u \in U, g \in G,
$$


This definition can be extended by linearity, to give an isomorphism

$$
\varphi \mapsto \varphi^{\mathrm{op}}: \operatorname{Hom}_{\mathcal{C}}(G, H) \rightarrow \operatorname{Hom}_{\mathcal{C}}(H, G) .
$$

It is easy to check that $(\varphi \circ \psi)^{\mathrm{op}}=\psi^{\mathrm{op}} \circ \varphi^{\mathrm{op}}$, with obvious notation, and the functor

$$
\begin{aligned}
G & \mapsto G \\
\varphi & \mapsto \varphi^{\text {op }}
\end{aligned}
$$

is an equivalence of categories from $\mathcal{C}$ to the dual category, which restricts to an equivalence of $\mathcal{C}_{p}$ to its dual category.

Example 3.9. If $G$ is a finite group, and $(T, S)$ is a section of $G$, then

$$
\left(\operatorname{Indinf}_{T / S}^{G}\right)^{\text {op }} \cong \operatorname{Defres}_{T / S}^{G}
$$

as $(T / S, G)$-bisets.

Notation and Definition 3.10. If $F$ is a biset functor, the dual biset functor $F^{*}$ is defined by

$$
F^{*}(G)=\operatorname{Hom}_{\mathbb{Z}}(F(G), \mathbb{Z}),
$$

for a finite group $G$, and by

$$
F^{*}(\varphi)(\alpha)=\alpha \circ F\left(\varphi^{\mathrm{op}}\right),
$$

for any $\alpha \in F^{*}(G)$, any finite group $H$, and any $\varphi \in \operatorname{Hom}_{\mathcal{C}}(G, H)$. In particular, if $(T, S)$ is a section of $G$, if $\varphi \in F^{*}(G)$ and $u \in F(T / S)$, then

$$
\left(\operatorname{Defres}_{T / S}^{G} \varphi\right)(u)=\varphi\left(\operatorname{Indinf}_{T / S}^{G} u\right) .
$$

Similarly, if $\psi \in F^{*}(T / S)$ and $v \in F(G)$, then

$$
\left(\operatorname{Indinf}_{T / S}^{G} \psi\right)(v)=\psi\left(\operatorname{Defres}_{T / S}^{G} v\right) .
$$

3.11. Subfunctors. Let $F$ be a biset functor. A biset subfunctor $F^{\prime}$ of $F$ consists of the data of a subgroup $F^{\prime}(G)$ of $F(G)$, for each finite group $G$, such that

$$
F(\varphi)\left(F^{\prime}(G)\right) \subseteq F^{\prime}(H),
$$

for any finite groups $G$ and $H$, and any $\varphi \in \operatorname{Hom}_{\mathcal{C}}(G, H)$. In this case, the quotient biset functor $F / F^{\prime}$ is defined in the obvious way.

The category $\mathcal{F}$ of biset functors is an abelian category, where a sequence

$$
F_{1} \stackrel{a}{\longrightarrow} F_{2} \stackrel{b}{\longrightarrow} F_{3}
$$


is exact if and only if all the evaluations

$$
F_{1}(G) \stackrel{a_{G}}{\longrightarrow} F_{2}(G) \stackrel{b_{G}}{\longrightarrow} F_{3}(G)
$$

at a particular finite group $G$, are exact sequences of abelian groups. In particular, the kernel and cokernel of a morphism $a: F^{\prime} \rightarrow F$ of biset functors are defined respectively by $(\operatorname{Ker} a)(G)=\operatorname{Ker} a_{G}$ and $(\operatorname{Coker} a)(G)=\operatorname{Coker} a_{G}$ for any finite group $G$.

Let $F$ be a biset functor. Let $\mathscr{H}$ be a set of finite groups, and let $S_{H}$ be a subset of $F(H)$, for any $H \in \mathcal{H}$. The subfunctor $\left\langle S_{H}\right\rangle_{H \in \mathscr{H}}$ of $F$ generated by these data is the intersection of the family of all subfunctors $F^{\prime}$ of $F$ such that $F^{\prime}(H) \supseteq S_{H}$, for any $H \in \mathscr{H}$. It is easy to see that for any finite group $G$

$$
\left\langle S_{H}\right\rangle_{H \in \mathscr{H}}(G)=\sum_{H \in \mathscr{H}} \operatorname{Hom}_{\mathcal{C}}(H, G)\left(S_{H}\right)
$$

is the subgroup of $F(G)$ generated by all images of elements of the sets $S_{H}$ by morphisms $H \rightarrow G$ in $\mathcal{C}$, for $H \in \mathscr{H}$.

The definitions and properties exposed in this subsection have obvious analogues for $p$-biset functors: the category $\mathcal{F}_{p}$ is an abelian category, where kernels and cokernels are defined "pointwise". The notion of subfunctor generated by some data extends trivially to the case of $p$-biset functors, by replacing the category $\mathcal{C}$ with $\mathcal{C}_{p}$.

3.12. Some idempotents in $\operatorname{End}_{\mathcal{C}}(G)$. Let $G$ be a finite group, and let $N \unlhd G$. Then it is clear from the definitions that

$$
\operatorname{Def}_{G / N}^{G} \circ \operatorname{Inf}_{G / N}^{G}=(G / N) \times{ }_{G}(G / N)=\operatorname{Id}_{G / N} .
$$

It follows that the composition $j_{N}^{G}=\operatorname{Inf}_{G / N}^{G} \circ \operatorname{Def}_{G / N}^{G}$ is an idempotent in $\operatorname{End}_{\mathcal{C}}(G)$. Moreover, if $M$ and $N$ are normal subgroups of $G$, then $j_{N}^{G} \circ j_{M}^{G}=j_{N M}^{G}$. Moreover $j_{1}^{G}=\operatorname{Id}_{G}$.

Lemma 3.14 ([9], Lemma 2.5). If $N \unlhd G$, define $f_{N}^{G} \in \operatorname{End}_{\mathcal{C}}(G)$ by

$$
f_{N}^{G}=\sum_{\substack{M \unlhd G \\ N \subseteq M}} \mu_{\unlhd G}(N, M) j_{M}^{G},
$$

where $\mu_{\unlhd G}$ denotes the Möbius function of the poset of normal subgroups of $G$. Then the elements $f_{N}^{G}$, for $N \unlhd G$, are orthogonal idempotents of $\operatorname{End}_{\mathcal{C}}(G)$, and their sum is equal to $\operatorname{Id}_{G}$. 
Moreover, it is easy to check from the definition that for $N \unlhd G$,

$$
f_{N}^{G}=\operatorname{Inf}_{G / N}^{G} \circ f_{1}^{G / N} \circ \operatorname{Def}_{G / N}^{G},
$$

and

$$
j_{N}^{G}=\operatorname{Inf}_{G / N}^{G} \circ \operatorname{Def}_{G / N}^{G}=\sum_{\substack{M \unlhd G \\ M \supseteq N}} f_{M}^{G} .
$$

Lemma 3.16. If $N$ is a non trivial normal subgroup of $G$, then

$$
f_{\mathbf{1}}^{G} \circ \operatorname{Inf}_{G / N}^{G}=0 \text { and } \operatorname{Def}_{G / N}^{G} \circ f_{1}^{G}=0 .
$$

Proof. Indeed by 3.15

$$
\begin{aligned}
f_{\mathbf{1}}^{G} \circ \operatorname{Inf}_{G / N}^{G} & =f_{\mathbf{1}}^{G} \circ \operatorname{Inf}_{G / N}^{G} \circ \operatorname{Def}_{G / N}^{G} \circ \operatorname{Inf}_{G / N}^{G} \\
& =\sum_{\substack{M \unlhd G \\
M \supseteq N}} f_{\mathbf{1}}^{G} f_{M}^{G} \operatorname{Inf}_{G / N}^{G}=0,
\end{aligned}
$$

since $M \neq \mathbf{1}$ when $M \supseteq N$. The other equality of the lemma follows by taking opposite bisets.

Remark 3.17. It was also shown in Section 2.7 of [9] that if $P$ is a $p$-group, then

$$
f_{\mathbf{1}}^{P}=\sum_{N \subseteq \Omega_{1} Z(P)} \mu(\mathbf{1}, N) P / N
$$

where $\mu$ is the Möbius function of the poset of subgroups of $N$, and $\Omega_{1} Z(P)$ is the subgroup of the centre of $P$ consisting of elements of order at most $p$.

Notation and Definition 3.18. If $F$ is a biset functor, and if $G$ is a finite group, then the idempotent $f_{\mathbf{1}}^{G}$ of $\operatorname{End}_{\mathcal{C}}(G)$ acts on $F(G)$. Its image

$$
\partial F(G)=f_{1}^{G} F(G)
$$

is a direct summand of $F(G)$ as $\mathbb{Z}$-module: it will be called the set of faithful elements of $F(G)$.

The reason for this name is that any element $u \in F(G)$ which is inflated from a proper quotient of $G$ is such that $F\left(f_{\mathbf{1}}^{G}\right) u=0$. From Lemma 3.16, it is also clear that

$$
\partial F(G)=\bigcap_{\mathbf{1} \neq N \unlhd G} \operatorname{Ker}^{\operatorname{Def}_{G / N}^{G}}
$$




\section{Genetic subgroups and rational $p$-biset functors}

Definition 4.1. A finite $p$-group $P$ has normal $p$-rank 1 if every abelian normal subgroup of $P$ is cyclic.

These groups have been classified long ago (see Theorem 4.10 of Chapter 5 of [17]): if $p \neq 2$, they are the cyclic $p$-groups, and if $p=2$, they are the cyclic 2-groups, the generalized quaternion 2-groups, the dihedral 2-groups of order at least 16, and the semi-dihedral 2-groups (of order at least 16).

The following definitions are essentially taken from Section 2 of [10]:

Notation and Definition 4.2. Let $P$ be a finite $p$-group. If $Q$ is a subgroup of $P$, denote by $Z_{P}(Q)$ the subgroup of $N_{P}(Q)$ defined by

$$
Z_{P}(Q) / Q=Z\left(N_{P}(Q) / Q\right) .
$$

A subgroup $Q$ of $P$ is called genetic if it satisfies the following two conditions:

(1) The group $N_{P}(Q) / Q$ has normal $p$-rank 1 .

(2) If $x \in P$, then $Q^{x} \cap Z_{P}(Q) \subseteq Q$ if and only if $Q^{x}=Q$.

Two genetic subgroups $Q$ and $R$ are said to be linked modulo $P$ (notation $Q-{ }_{P} R$ ), if there exist elements $x$ and $y$ in $P$ such that $Q^{x} \cap Z_{P}(R) \subseteq R$ and $R^{y} \cap Z_{P}(Q) \subseteq Q$.

This relation is an equivalence relation on the set of genetic subgroups of $P$. The set of equivalence classes is in one to one correspondence with the set of isomorphism classes of rational irreducible representations of $P$, by Section 1.7 and Proposition 4.4 of [9]. A genetic basis of $P$ is a set of representatives of these equivalences classes.

If $V$ is an irreducible representation of $P$, then the type of $V$ is the isomorphism class of the group $N_{P}(Q) / Q$, where $Q$ is a genetic subgroup of $P$ in the equivalence class corresponding to $V$ by the above bijection.

Remark 4.3. The definition of the relation $-P$ given here is different from Definition 2.9 of [10], but it is equivalent to it, by Lemma 4.5 of [9].

Example 4.4. If $P$ is an abelian $p$-group, then it follows from the definition that a subgroup $Q$ of $P$ is genetic if and only if the group $P / Q$ is cyclic. Moreover, if $Q$ and $R$ are genetic subgroups of $P$, then $Q-P R$ if and only if $Q=R$. So if $P$ is abelian, there is a unique genetic basis of $P$, consisting of all subgroups $Q$ of $P$ such that $P / Q$ is cyclic.

The following is Theorem 3.2 of [9], in a slightly different form: 
Theorem 4.5. Let $P$ be a finite $p$-group, and $q$ be a genetic basis of $P$. Let $F$ be a p-biset functor. Then the map

$$
\tau_{g}=\bigoplus_{Q \in \mathcal{G}} \operatorname{Indinf}_{N_{P}(Q) / Q}^{P}: \bigoplus_{Q \in \mathcal{G}} \partial F\left(N_{P}(Q) / Q\right) \rightarrow F(P)
$$

is split injective.

Remark 4.6. There are two differences with the initial statement of Theorem 3.2 of [9]: here I use genetic subgroups instead of genetic sections, because these two notions are equivalent by Proposition 4.4 of [9]. Also the definition of the map $\tau_{g}$ is apparently different: with the notation of [9], the map $\tau_{g}$ is the sum of the maps $F\left(a_{Q}\right)$, where $a_{Q}$ is the trivial $(P, P / P)$-biset if $Q=P$, and $a_{Q}$ is the virtual $\left(P, N_{P}(Q) / Q\right)$ )-biset $P / Q-P / \hat{Q}$ if $Q \neq P$, where $\hat{Q}$ is the unique subgroup of $Z_{P}(Q)$ containing $Q$, and such that $|\hat{Q}: Q|=p$. But it is easy to see that the restriction of the map $F(P / \hat{Q})$ to $\partial F\left(N_{P}(Q) / Q\right)$ is actually 0 . Moreover, the map $F(P / Q)$ is equal to $\operatorname{Indinf}_{N_{P}(Q) / Q}^{P}$. So in fact, the above map $I_{g}$ is the same as the one defined in Theorem 3.2 of [9].

Definition 4.7. A $p$-biset functor $F$ is called rational if for any finite $p$-group $P$, the map $\tau_{g}$ is an isomorphism, for some genetic basis $g$ of $P$.

Lemma 7.3 of [9] shows that this condition does not depend on the genetic basis $g$ of $P$. In other words, the word some in Definition 4.7 can be replaced by the word any.

Remark 4.8. This definition and the use of the word rational were first motivated by the case of the functor $R_{\mathbb{Q}}$, which is the typical example of a rational $p$-biset functor (see Example 7.2 of [9] for details). More generally, the definition of a rational $p$-biset functor shows that the evaluations of such a functor can be computed from its values at $p$-groups of normal $p$-rank 1 , which are generally easy to obtain.

Remark 4.9. Another useful property of this particular class of functors is that the full subcategory of the category $\mathcal{F}_{p}$ whose objects are rational $p$-biset functors is a Serre subcategory: in other words, if $F^{\prime}$ is a subfunctor of the $p$-biset functor $F$, then $F$ is rational if and only if $F^{\prime}$ and $F / F^{\prime}$ are rational. Moreover, any dual functor of a rational biset functor is a rational biset functor (see Proposition 7.4 of [9] for details about these results).

\section{The functor of units of the Burnside ring}

If $G$ and $H$ are finite groups, and if $U$ is a finite $(H, G)$-biset, recall that $U^{\text {op }}$ denotes the $(G, H)$-biset obtained from $U$ by reversing the actions. If $X$ is a finite $G$-set, then 
$T_{U}(X)=\operatorname{Hom}_{G}\left(U^{\mathrm{op}}, X\right)$ is a finite $H$-set. The correspondence $X \mapsto T_{U}(X)$ can be extended to a correspondence $T_{U}: B(G) \rightarrow B(H)$, which is multiplicative (i.e. $T_{U}(a b)=T_{U}(a) T_{U}(b)$ for any $a, b \in B(G)$ ), and preserves identity elements (i.e. $\left.T_{U}(G / G)=H / H\right)$. This extension to $B(G)$ can be built by different means: the following is described in Section 4.1 of [5], and it is similar to a construction given by T. tom Dieck ([23]). It uses $G$-posets and Lefschetz invariants, whose definition is due to J. Thévenaz ([22]):

Definition 5.1. Let $G$ be a finite group. A finite $G$-poset $X$ is a finite $G$-set $X$, partially ordered by a $G$-invariant relation $\leq$ (i.e. for any $g \in G$ and any $x, y \in X$, if $x \leq y$, then $g x \leq g y$ ).

The Lefschetz invariant $\Lambda_{X}$ of a finite $G$-poset $X$ is the element of $B(G)$ defined by

$$
\Lambda_{X}=\sum_{s \in[G \backslash S d(X)]}(-1)^{\operatorname{dim}(s)} G / G_{s},
$$

where $[G \backslash S d(X)]$ is a set of representatives of $G$-orbits on the set $S d(X)$ of linearly ordered subsets of $X$, where $\operatorname{dim}(s)=|s|-1$ and $G_{s}$ is the stabilizer of s in $G$.

Remark 5.2. The idea of considering this kind of "Euler characteristic" is already mentioned informally by T. tom Dieck ([23], Chapter 1), who uses this terminology because of the following: if $H$ is a subgroup of $G$, then

$$
\left|\left(\Lambda_{X}\right)^{H}\right|=\chi\left(X^{H}\right)=\sum_{s \in S d\left(X^{H}\right)}(-1)^{\operatorname{dim}(s)}
$$

is the Euler-Poincaré characteristic of the poset $X^{H}$.

Example 5.3. Let $X$ be a $G$-set. Consider $X$ as a $G$-poset for the equality relation (i.e. $x \leq y$ in $X$ if and only if $x=y$ ). Then $\Lambda_{X}=X$ in $B(G)$, and $\chi\left(X^{H}\right)=\left|X^{H}\right|$ for any subgroup $H$ of $G$.

If $a$ is an element of $B(G)$, it is easy to show that there exists a finite $G$-poset $X$ such that $a$ is equal to the Lefschetz invariant $\Lambda_{X}$ ([2], Lemme 2). Now $\operatorname{Hom}_{G}\left(U^{\mathrm{op}}, X\right)$ has a natural structure of $H$-poset, and one can set $T_{U}(a)=\Lambda_{\mathrm{Hom}_{G}\left(U^{\mathrm{op}}, X\right)}$. It is an element of $B(H)$, which does not depend of the choice of the poset $X$ such that $a=\Lambda_{X}$, because with Notation 3.6 and Lemma 3.7, for any subgroup $T$ of $H$ the Euler-Poincaré characteristic $\chi\left(\operatorname{Hom}_{G}\left(U^{\mathrm{op}}, X\right)^{T}\right)$ can be computed by

$$
\chi\left(\operatorname{Hom}_{G}\left(U^{\mathrm{op}}, X\right)^{T}\right)=\prod_{u \in T \backslash U / G} \chi\left(X^{T^{u}}\right),
$$

and the latter only depends on the element $\Lambda_{X}$ of $B(G)$. This shows that $T_{U}(a)$ is well defined, and can be computed with the following formula: 
Lemma 5.4. Let $G$ and $H$ be finite groups, and let $U$ be a finite $(H, G)$-biset. Then for any $a \in B(G)$ and any subgroup $T$ of $H$

$$
\left|T_{U}(a)^{T}\right|=\prod_{u \in T \backslash U / G}\left|a^{T^{u}}\right| .
$$

It follows in particular that $T_{U}\left(B^{\times}(G)\right) \subseteq B^{\times}(H)$. Moreover, it is easy to check that $T_{U}=T_{U^{\prime}}$ if $U$ and $U^{\prime}$ are isomorphic $(H, G)$-bisets, and that $T_{U_{1} \sqcup U_{2}}(a)=$ $T_{U_{1}}(a) T_{U_{2}}(a)$ for any $(H, G)$-bisets $U_{1}$ and $U_{2}$, and any $a \in B(G)$.

It follows that there is a well defined bilinear pairing

$$
B\left(H \times G^{\mathrm{op}}\right) \times B^{\times}(G) \rightarrow B^{\times}(H),
$$

extending the correspondence $(U, a) \mapsto T_{U}(a)$. If $f \in B\left(H \times G^{\mathrm{op}}\right)$ (i.e. if $f$ is a virtual $(H, G)$-biset), the corresponding group homomorphism $B^{\times}(G) \rightarrow B^{\times}(H)$ will be denoted by $B^{\times}(f)$.

Now let $K$ be a third group, and $V$ be a finite $(K, H)$-set. If $X$ is a finite $G$-set, there is a canonical isomorphism of $K$-sets

$$
\operatorname{Hom}_{H}\left(V^{\mathrm{op}}, \operatorname{Hom}_{G}\left(U^{\mathrm{op}}, X\right)\right) \cong \operatorname{Hom}_{G}\left(\left(V \times_{H} U\right)^{\mathrm{op}}, X\right),
$$

showing that $T_{V} \circ T_{U}=T_{V \times{ }_{H} U}$.

It follows more generally that $B^{\times}(g) \circ B^{\times}(f)=B^{\times}\left(g \times{ }_{H} f\right)$ for any $g \in$ $B\left(K \times H^{\mathrm{op}}\right)$ and any $f \in B\left(H \times G^{\mathrm{op}}\right)$. Finally this shows:

Proposition 5.5. The correspondence sending a finite group $G$ to $B^{\times}(G)$, and a homomorphism $f$ in $\mathcal{C}$ to $B^{\times}(f)$, is a biset functor.

Remark 5.6. The restriction and inflation maps for the functor $B^{\times}$coincide with the usual ones for the functor $B$. The deflation map $\operatorname{Def}_{G / N}^{G}$ corresponds to taking fixed points under $N$ (so it does not coincide with the usual deflation map for $B$, which consist in taking orbits under $N$ ).

Similarly, if $H$ is a subgroup of $G$, the induction map from $H$ to $G$ for the functor $B^{\times}$is the tensor induction $\operatorname{Ten}_{H}^{G}$, and it is different from the induction map $\operatorname{Ind}_{H}^{G}$ for the Burnside functor $B$.

\section{Faithful elements in $B^{\times}(G)$}

The following lemma ([19], Lemma 2.8) is a key tool in this section:

Lemma 6.1 (Matsuda-Miyata). Let $G$ be a finite group, and $u \in B^{\times}(G)$. If $(T, S)$ is a section of $G$ such that $T / S$ is an odd order p-group, then $\left|u^{S}\right|=\left|u^{T}\right|$. 
Proof. Indeed, the element $u$ can be written as the difference $X-Y$ of two $G$ sets $X$ and $Y$. Now $u^{S}=X^{S}-Y^{S}$ in $B\left(N_{G}(S) / S\right)$. Moreover the $p$-group $T / S$ acts on $X^{S}$, and the set of fixed points under this action is $X^{T}$. It is well known that $\left|X^{S}\right| \equiv\left|\left(X^{S}\right)^{T / S}\right|$ modulo $p$. Of course, a similar congruence holds for $\left|Y^{S}\right|$, showing that $\left|u^{S}\right|$ and $\left|u^{T}\right|$ are congruent modulo $p$. But $\left|u^{S}\right|$ and $\left|u^{T}\right|$ are equal to \pm 1 if $u \in B^{\times}(G)$. Since $p \geq 3$, this forces $\left|u^{S}\right|=\left|u^{T}\right|$.

In the following lemma, Assertion 2 is a refinement of a result of Matsuda ([18]): in the case of a nilpotent group $G$, it gives an explicit isomorphism between $B^{\times}(G)$ and $B^{\times}\left(G_{2}\right)$, where $G_{2}$ is a Sylow 2-subgroup of $G$. The most general form of the argument requires the following notation:

Notation 6.2. If $G$ is a finite group, denote by $I(G)$ the subgroup defined by

$$
I(G)=\bigcap_{H \subseteq G} N_{G}(H),
$$

where the intersection runs over the set of all subgroups $H$ of $G$.

The subgroup $I(G)$ has been considered in particular by Beidleman, Heineken and Newell ([1]), who call it the norm of $G$. Obviously $I(G)$ contains the centre of $G$. Moreover, every subgroup of $I(G)$ is normal in $I(G)$, so $I(G)$ is nilpotent. Though it is not necessary for the following proof, it should be noted that by a theorem of Dedekind ([14]), the odd order part of $I(G)$ is abelian.

Lemma 6.3. (1) Let $G$ be a finite group, and let $Z$ be a normal subgroup of odd order of $G$, contained in $I(G)$ (e.g. a central subgroup of odd order of $G)$. Then the inflation map

$$
\operatorname{Inf}_{G / Z}^{G}: B^{\times}(G / Z) \rightarrow B^{\times}(G)
$$

is an isomorphism.

(2) If $G$ is nilpotent, then $G \cong G_{\text {odd }} \times G_{2}$, where $G_{\text {odd }}$ is the subgroup of elements of odd order of $G$, and $G_{2}$ is the unique Sylow 2-subgroup of $G$. Then the group isomorphism $G / G_{\text {odd }} \cong G_{2}$, composed with the inflation map $\operatorname{Inf}_{G / G_{\text {odd }}}^{G}$ induces an isomorphism $B^{\times}\left(G_{2}\right) \rightarrow B^{\times}(G)$.

(3) In particular, if $G$ is an odd order p-group, then $B^{\times}(G)=\left\{ \pm 1_{B}\right\}$.

Proof. For Assertion 1, consider an element $u$ of $B^{\times}(G)$. If $H$ is a subgroup of $G$, then $\left|u^{H}\right|=\left|u^{H Z}\right|$ : indeed, the group $Z$ normalizes $H$, and it is nilpotent, so it has a filtration

$$
Z=Z_{0} \supset Z_{1} \supset \cdots \supset Z_{n}=\mathbf{1}
$$

by (normal) subgroups $Z_{i}$, for $i=0, \ldots, n$, such that each factor group $Z_{i} / Z_{i+1}$ is an odd prime order $p_{i}$-group, for some $p_{i}$, for $i=0, \ldots, n-1$. The equality $\left|u^{H}\right|=$ 
$\left|u^{H Z}\right|$ follows, by repeated application of Lemma 6.1 to the section $\left(H Z_{i}, H Z_{i+1}\right)$ of $G$.

Now consider the element $v=\operatorname{Inf}_{G / Z}^{G} \operatorname{Def}_{G / Z}^{G} u$. For any subgroup $H$ of $G$, one has that $\left|v^{H}\right|=\left|u^{H Z}\right|$. Hence $u=v$. In other words the map $\operatorname{Inf}_{G / Z}^{G} \operatorname{Def}_{G / Z}^{G}$ is the identity map of $B^{\times}(G)$. This shows Assertion 1 , since the map $\operatorname{Def}_{G / Z}^{G} \operatorname{Inf}_{G / Z}^{G}$ is always the identity map of $B^{\times}(G / Z)$, by 3.13 .

Assertion 2 follows easily by induction on the order of $G_{\text {odd }}$, using a subgroup $Z$ of odd order in the centre of $G$. Assertion 3 is the special case of Assertion 2, when $G_{2}=\mathbf{1}$.

Remark 6.4. So in the sequel, when considering $p$-groups, the only really non-trivial case will occur for $p=2$. However, some statements will be given for arbitrary $p$ groups.

The following result uses the same kind of elementary argument that was used in Lemma 6.3, the main difference being that it also requires Feit-Thompson's Theorem. It is a slight generalization of a theorem of Matsuda and Miyata:

Proposition 6.5. Let $G$ be a finite group, and $N$ be a normal subgroup of odd index in $G$. Then the group $G / N$ acts on $B^{\times}(N)$, and the maps $\operatorname{Res}_{N}^{G}$ and $\operatorname{Ten}_{N}^{G}$ induce mutual inverse isomorphisms between $B^{\times}(G)$ and $B^{\times}(N)^{G / N}$.

Proof. First the group $G$ acts on $N$ by conjugation, hence it acts on $B(N)$ by ring automorphisms, so it acts on $B^{\times}(N)$ by group automorphisms. Moreover, the action of $N$ on $B(N)$ is trivial, so $G / N$ acts on $B^{\times}(N)$.

It is also clear that $\operatorname{Res}_{N}^{G} B^{\times}(G) \subseteq B^{\times}(N)^{G / N}$ : indeed, if $u \in B(G)$ and $g \in G$, then

$$
{ }^{g}\left(\operatorname{Res}_{N}^{G} u\right)=\operatorname{Res}_{N}^{G}\left({ }^{g} u\right)=\operatorname{Res}_{N}^{G} u .
$$

Now for any subgroup $H$ of $G$, by Lemma 5.4

$$
\begin{aligned}
\left|\left(\operatorname{Ten}_{N}^{G} \operatorname{Res}_{N}^{G} u\right)^{H}\right| & =\prod_{g \in H N \backslash G}\left|\left(\operatorname{Res}_{N}^{G} u\right)^{N \cap g} H\right| \\
& =\prod_{g \in H N \backslash G}\left|u^{N \cap^{g} H}\right| \\
& =\prod_{g \in H N \backslash G}\left|u^{N \cap H}\right| \quad\left(\text { since } N \cap \cap^{g} H=g(N \cap H)\right) \\
& =\left|u^{N \cap H}\right|^{|G: H N|}=\left|u^{N \cap H}\right|
\end{aligned}
$$

since $|G: H N|$ is odd. Now the group $H /(H \cap N) \cong H N / N$ has odd order, hence it is solvable, by Feit-Thompson's Theorem, and as in Lemma 6.3, an easy induction 
argument, using Lemma 6.1, shows that $\left|u^{N \cap H}\right|=\left|u^{H}\right|$. So $\left|\left(\operatorname{Ten}_{N}^{G} \operatorname{Res}_{N}^{G} u\right)^{H}\right|=$ $\left|u^{H}\right|$ for any subgroup $H$ of $G$, and $\operatorname{Ten}_{N}^{G} \operatorname{Res}_{N}^{G}$ is the identity map of $B^{\times}(G)$.

Now if $v \in B^{\times}(N)^{G / N}$, the element $\operatorname{Res}_{N}^{G} \operatorname{Ten}_{N}^{G} v$ is obtained from $v$ by applying the $(N, N)$-biset $G$. Since $N \unlhd G$, this biset is isomorphic to the disjoint union of the classes $N x$, for $x \in G / N$, each such class being viewed as an $(N, N)$-biset by left and right multiplication. Clearly this biset $N x$ is isomorphic to the $(N, N)$-biset associated to the automorphism $n \mapsto{ }^{x} n$ of $N$. Thus

$$
\operatorname{Res}_{N}^{G} \operatorname{Ten}_{N}^{G} v=\sum_{x \in G / N}{ }^{x} v=|G: N| v=v,
$$

since $v$ is invariant under the action of $G / N$, and since $|G / N|$ is odd and $2 v=0$. Hence $\operatorname{Res}_{N}^{G} \operatorname{Ten}_{N}^{G}$ is the identity map of $B^{\times}(N)^{G / N}$, as was to be shown.

Remark 6.6. Matsuda and Miyata ([19], Theorem B iii) proved that the restriction $\operatorname{Res}_{N}^{G}: B^{\times}(G) \rightarrow B^{\times}(N)^{G / N}$ is an isomorphism, under the additional hypothesis that the group $G$ is isomorphic to the semi-direct product of $N$ and $G / N$.

Notation 6.7. If $G$ is a finite group, denote by $F_{G}$ the set of subgroups $H$ of $G$ such that $H \cap Z(G)=\mathbf{1}$, and set $\left[F_{G}\right]=F_{G} \cap\left[s_{G}\right]$.

Lemma 6.8. Let $G$ be a finite group. If $|Z(G)|>2$, then $\partial B^{\times}(G)$ is trivial.

Proof. Recall that if $H \in\left[s_{G}\right]$, then the corresponding primitive idempotent $e_{H}^{G}$ of $\mathbb{Q} B(G)$ has the property that $a e_{H}^{G}=\left|a^{H}\right| e_{H}^{G}$, for any $a \in B(G)$. Thus

$$
a=\sum_{H \in\left[s_{G}\right]}\left|a^{H}\right| e_{H}^{G}
$$

Now let $a \in \partial B^{\times}(G)$. Then $\operatorname{Def}_{G / N}^{G} a$ is the identity element of $B^{\times}(G / N)$, for any non-trivial normal subgroup $N$ of $G$. Now suppose that $H$ is a subgroup of $G$ containing $N$. Then

$\left|a^{H}\right|=\left|\operatorname{Defres}_{N_{G}(H) / H}^{G} a\right|=\left|\operatorname{Iso}_{N_{G / N}(H / N) /(H / N)}^{N_{G}(H) / H} \operatorname{Defres}_{N_{G / N}(H / N)}^{G / N} \operatorname{Def}_{G / N}^{G} a\right|=1$.

In particular $\left|a^{H}\right|=1$ if $H \cap Z(G) \neq \mathbf{1}$. It follows that there exists a subset $A$ of $\left[F_{G}\right]$ such that

$$
a=G / G-2 \sum_{H \in A} e_{H}^{G} .
$$

If $A \neq \emptyset$, i.e. if $a \neq G / G$, let $L$ be a maximal element of $A$. Then $L \neq G$, because $Z(G) \neq 1$. The coefficient of $G / L$ in the expression of $a$ in the canonical basis of $B(G)$ is equal to

$$
-2 \frac{|L| \mu(L, L)}{\left|N_{G}(L)\right|}=-2 \frac{|L|}{\left|N_{G}(L)\right|} .
$$


This is moreover an integer, since $a \in B^{\times}(G)$. It follows that $\left|N_{G}(L): L\right|$ is equal to 1 or 2. But since $L \cap Z(G)=\mathbf{1}$, the group $Z(G)$ embeds into the group $N_{G}(L) / L$. Hence $\left|N_{G}(L): L\right| \geq 3$, and this contradiction shows that $A=\emptyset$, thus $a=G / G$.

Lemma 6.9. Let $P$ be a finite 2-group, of order at least 4 , and suppose that the maximal elements of $F_{P}$ have order 2 . If $|P| \geq 2\left|F_{P}\right|$, then $\partial B^{\times}(P)$ is trivial.

Proof. Let $a \in \partial B^{\times}(P)$. By the argument of the previous proof, there exists a subset $A$ of $\left[F_{P}\right]$ such that

$$
a=P / P-2 \sum_{H \in A} e_{H}^{P} .
$$

The hypothesis implies that $\mu(\mathbf{1}, H)=-1$ for any non-trivial element $H$ of $\left[F_{P}\right]$. Now if $\mathbf{1} \in A$, the coefficient of $P / 1$ in the expression of $a$ in the canonical basis of $B(P)$ is equal to

$$
-2 \frac{1}{|P|}+2 \sum_{H \in A-\{\mathbf{1}\}} \frac{1}{\left|N_{P}(H)\right|}=-2 \frac{1}{|P|}+2 \sum_{H \in \bar{A}-\{\mathbf{1}\}} \frac{1}{|P|}=\frac{-4+2|\bar{A}|}{|P|},
$$

where $\bar{A}$ is the set of subgroups of $P$ which are conjugate to some element of $A$. This coefficient is an integer if $a \in B(P)$, so $|P|$ divides $2|\bar{A}|-4$. But $|\bar{A}|$ is always odd, since the trivial subgroup is the only normal subgroup of $P$ which is in $\bar{A}$ in this case. Thus $2|\bar{A}|-4$ is congruent to 2 modulo 4 , and cannot be divisible by $|P|$, since $|P| \geq 4$.

So $\mathbf{1} \notin A$, and the coefficient of $P / 1$ in the expression of $a$ is equal to

$$
2 \sum_{H \in A} \frac{1}{\left|N_{P}(H)\right|}=\frac{2|\bar{A}|}{|P|} .
$$

Now this is an integer, so $2|\bar{A}|$ is congruent to 0 or 1 modulo the order of $P$, which is even since $|P| \geq 2\left|F_{P}\right| \geq 2$. Thus $\mathbf{1} \notin A$, and $2|\bar{A}|$ is a multiple of $|P|$. But $2|\bar{A}|<2\left|F_{P}\right|$ since $\mathbf{1} \notin A$. So if $2\left|F_{P}\right| \leq|P|$, it follows that $\bar{A}$ is empty, and $A$ is empty. Hence $a=P / P$, as was to be shown.

Corollary 6.10. Let $P$ be a finite 2-group. Then the group $\partial B^{\times}(P)$ is trivial in each of the following cases:

(1) $P$ is abelian of order at least 3.

(2) $P$ is generalized quaternion or semi-dihedral.

Remark 6.11. Case 1 follows easily from Matsuda's Theorem 2.11. Case 2 follows from Lemma 4.6 of Yalçın ([24]). 
Proof. Case 1 follows from Lemma 6.8. In Case 2, if $P$ is generalized quaternion, then $F_{P}=\{\mathbf{1}\}$, thus $|P| \geq 2\left|F_{P}\right|$. And if $P$ is semidihedral, then there is a unique conjugacy class of non-trivial subgroups $H$ of $P$ such that $H \cap Z(P)=1$. Such a group has order 2 , and $N_{P}(H)=H Z(P)$ has order 4 . Thus $\left|F_{P}\right|=1+\frac{|P|}{4}$, and $|P| \geq 2\left|F_{P}\right|$ also in this case.

Corollary 6.12 (Yalçın [24], Lemma 4.6 and Lemma 5.2). Let $P$ be a p-group of normal p-rank 1 . Then $\partial B^{\times}(P)$ is trivial, except if $P$ is

- the trivial group, and $\partial B^{\times}(P)$ is the group of order 2 generated by $v_{P}=-P / P$;

- cyclic of order 2 , and $\partial B^{\times}(P)$ is the group of order 2 generated by

$$
v_{P}=P / P-P / \mathbf{1}
$$

- dihedral of order at least 16 , and then $\partial B^{\times}(P)$ is the group of order 2 generated by the element

$$
v_{P}=P / P+P / 1-P / I-P / J,
$$

where $I$ and $J$ are non-central subgroups of order 2 of $P$, not conjugate in $P$.

Proof. Recall that the $p$-groups of normal $p$-rank 1 (see Definition 4.1) are the cyclic groups if $p \neq 2$, or the cyclic groups, the generalized quaternion groups, and the dihedral or semi-dihedral groups of order at least 16 if $p=2$.

Lemma 6.3 and Lemma 6.8 show that $\partial B^{\times}(P)$ is trivial, when $P$ has normal $p$-rank 1, except possibly if $P$ is trivial, cyclic of order 2 , or dihedral.

Now if $P$ is trivial, then obviously $B(P)=\mathbb{Z}$, so $B^{\times}(P)=\partial B^{\times}(P)=\{ \pm P / P\}$. If $P$ has order 2 , then clearly $B^{\times}(P)$ consists of $\pm P / P$ and $\pm(P / P-P / \mathbf{1})$, and $\partial B^{\times}(P)=\{P / P, P / P-P / \mathbf{1}\}$. Finally, if $P$ is dihedral, the set $F_{P}$ consists of the trivial group, and of two conjugacy classes of subgroups $H$ of order 2 of $P$, and $N_{P}(H)=H Z$ for each of these, where $Z$ is the centre of $P$. Thus

$$
\left|F_{P}\right|=1+2 \frac{|P|}{4}=1+\frac{|P|}{2} .
$$

Now with the notation of the proof of Lemma 6.9, one has that $2|\bar{A}| \equiv 0(|P|)$, and $2|\bar{A}|<2\left|F_{P}\right|=2+|P|$. So either $A=\emptyset$, and in this case $a=P / P$, or $2|\bar{A}|=|P|$, which means that $\bar{A}$ is the whole set of non-trivial elements of $F_{P}$. In this case

$$
a=P / P-2\left(e_{I}^{P}+e_{J}^{P}\right),
$$

where $I$ and $J$ are non-central subgroups of order 2 of $P$, not conjugate in $P$. It is then easy to check that

$$
a=P / P+P / \mathbf{1}-(P / I+P / J),
$$

so $a$ is indeed in $B(P)$, hence in $B^{\times}(P)$. Moreover $\operatorname{Def}_{P / Z}^{P} a$ is the identity element of $B^{\times}(P / Z)$, so $a=f_{1}^{P} a$, and $a \in \partial B^{\times}(P)$. This completes the proof. 


\section{A morphism of biset functors}

If $k$ is any commutative ring, there is an obvious isomorphism of biset functor from $k B^{*}=k \otimes_{\mathbb{Z}} B^{*}$ to $\operatorname{Hom}(B, k)$, sending the element $\alpha=\sum_{i} \alpha_{i} \otimes \psi_{i}$, where $\alpha_{i} \in k$ and $\psi_{i} \in B^{*}(G)$, to the linear form $\tilde{\alpha}: B(G) \rightarrow k$ defined by $\tilde{\alpha}(G / H)=$ $\sum_{i} \psi_{i}(G / H) \alpha_{i}$.

Notation 7.1. Let $\{ \pm 1\}=\mathbb{Z}^{\times}$be the group of units of the ring $\mathbb{Z}$. The unique group isomorphism from $\{ \pm 1\}$ to $\mathbb{Z} / 2 \mathbb{Z}$ will be denoted by $u \mapsto u_{+}$.

If $G$ is a finite group, and if $a \in B^{\times}(G)$, then recall that for each subgroup $S$ of $G$, the integer $\left|a^{S}\right|$ is equal to \pm 1 . Define a map $\varepsilon_{G}: B^{\times}(G) \rightarrow \mathbb{F}_{2} B^{*}(G)$ by setting $\varepsilon_{G}(a)(G / S)=\left|a^{S}\right|_{+}$, for any $a \in B^{\times}(G)$ and any subgroup $S$ of $G$.

Proposition 7.2. The maps $\varepsilon_{G}$ define an injective morphism of biset functors

$$
\varepsilon: B^{\times} \rightarrow \mathbb{F}_{2} B^{*} .
$$

Proof. The injectivity of the map $\varepsilon_{G}$ is obvious. Now let $G$ and $H$ be finite groups, and let $U$ be a finite $(H, G)$-biset. Also denote by $U$ the corresponding element of $B\left(H \times G^{\mathrm{op}}\right)$. If $a \in B^{\times}(G)$, and if $T$ is a subgroup of $H$, then by Lemma 5.4

$$
\left|B^{\times}(U)(a)^{T}\right|=\prod_{u \in T \backslash U / G}\left|a^{T^{u}}\right| .
$$

Thus

$$
\begin{aligned}
\varepsilon_{H}\left(B^{\times}(U)(a)\right)(H / T) & =\left(\prod_{u \in T \backslash U / G} \mid a^{T^{u}}\right)_{+} \\
& =\sum_{u \in T \backslash U / G}\left|a^{T^{u}}\right|_{+} \\
& =\sum_{u \in T \backslash U / G} \varepsilon_{G}(a)\left(G / T^{u}\right) \\
& =\varepsilon_{G}(a)\left(U^{\mathrm{op}} / T\right) \quad(\text { by Lemma 3.7) } \\
& =\varepsilon_{G}(a)\left(U^{\mathrm{op}} \times_{H} H / T\right) \\
& =\mathbb{F}_{2} B^{*}(U)\left(\varepsilon_{G}(a)\right)(H / T)
\end{aligned}
$$

thus $\varepsilon_{H} \circ B^{\times}(U)=\mathbb{F}_{2} B^{*}(U) \circ \varepsilon_{G}$. Since both sides are additive with respect to $U$, the same equality holds when $U$ is an arbitrary element of $B\left(H \times G^{\mathrm{op}}\right)$, completing the proof. 


\section{Restriction to the case of $p$-groups}

The additional result that holds for finite $p$-groups (and not for arbitrary finite groups) is the Ritter-Segal theorem ([20], [21], see also [6]), which says that the natural transformation $B \rightarrow R_{\mathbb{Q}}$ of biset functors for $p$-groups, is surjective. By duality, it follows that the natural transformation $i: k R_{\mathbb{Q}}^{*} \rightarrow k B^{*}$ is injective, for any commutative ring $k$. The following gives a characterization of the image $i\left(k R_{\mathbb{Q}}^{*}\right)$ inside $k B^{*}$ :

Proposition 8.1. Let $p$ be a prime number, let $P$ be a p-group, let $k$ be a commutative ring. Then the element $\varphi \in k B^{*}(P)$ lies in $i\left(k R_{\mathbb{Q}}^{*}(P)\right)$ if and only if the element $\operatorname{Defres}_{T / S}^{P} \varphi$ lies in $i\left(k R_{\mathbb{Q}}^{*}(T / S)\right)$, for any section $T / S$ of $P$ which is

- elementary abelian of rank 2, or non-abelian of order $p^{3}$ and exponent $p$, if $p \neq 2$;

- elementary abelian of rank 2 , or dihedral of order at least 8 , if $p=2$.

Proof. Since the image of $k R_{\mathbb{Q}}^{*}$ is a subfunctor of $k B^{*}$, if $\varphi \in i\left(k R_{\mathbb{Q}}^{*}(P)\right)$, then $\operatorname{Defres}_{T / S}^{P} \varphi \in i\left(k R_{\mathbb{Q}}^{*}(T / S)\right)$, for any section $(T, S)$ of $P$.

Conversely, consider the exact sequence of biset functors over $p$-groups

$$
0 \rightarrow K \rightarrow B \rightarrow R_{Q} \rightarrow 0 .
$$

Every evaluation of this sequence at a particular $p$-group is a split exact sequence of (free) abelian groups. Hence by duality, for any ring $k$, there is an exact sequence

$$
0 \rightarrow k R_{\mathbb{Q}}^{*} \rightarrow k B^{*} \rightarrow k K^{*} \rightarrow 0 .
$$

With the identification $k B^{*} \cong \operatorname{Hom}_{\mathbb{Z}}(B, k)$, this means that if $P$ is a $p$-group, the element $\varphi \in k B^{*}(P)$ lies in $i\left(k R_{\mathbb{Q}}^{*}(P)\right)$ if and only if $\varphi(K(P))=0$. Now by Corollary 6.16 of [10], the group $K(P)$ is the set of linear combinations of elements of the form $\operatorname{Ind} \inf _{T / S}^{P} \theta(\kappa)$, where $T / S$ is a section of $P$, and $\theta$ is a group isomorphism from one of the group listed in the statement to $T / S$, and $\kappa$ is a specific element of $K(T / S)$ in each case. The proposition follows, because

$$
\varphi\left(\operatorname{Indinf}_{T / S}^{P} \theta(\kappa)\right)=\left(\operatorname{Defres}_{T / S}^{P} \varphi\right)(\theta(\kappa)),
$$

and this is zero if $\operatorname{Defres}_{T / S}^{P} \varphi$ lies in $i\left(k R_{\mathbb{Q}}^{*}(T / S)\right)$.

Theorem 8.2. Let $p$ be a prime number, and $P$ be a finite p-group. The image of the map $\varepsilon_{P}$ is contained in $i\left(\mathbb{F}_{2} R_{\mathbb{Q}}^{*}(P)\right)$.

Proof. The proof goes by induction on the order of $P$ : suppose that

$$
\varepsilon_{Q}\left(B^{\times}(Q)\right) \subseteq i_{Q}\left(\mathbb{F}_{2} R_{\mathbb{Q}}^{*}(Q)\right),
$$


for any $p$-group $Q$ with $|Q|<|P|$. Let $a \in B^{\times}(P)$, and let $T / S$ be any section of $P$. Since

$$
\operatorname{Defres}_{T / S}^{P} \varepsilon_{P}(a)=\varepsilon_{T / S} \operatorname{Defres}_{T / S}^{P} a,
$$

and since this lies in $i_{T / S}\left(\mathbb{F}_{2} R_{\mathbb{Q}}^{*}(T / S)\right)$ if $|T / S|<|P|$, by induction hypothesis, Proposition 8.1 shows that one can suppose that $P$ is elementary abelian of rank 2 or non-abelian of order $p^{3}$ and exponent $p$ if $p$ is odd, or that $P$ is elementary abelian of rank 2 or dihedral if $p=2$.

Now if $N$ is a normal subgroup of $P$, by 3.15 , one has that

$$
f_{N}^{P} \varepsilon_{P}(a)=\varepsilon_{P} f_{N}^{P}(a)=\operatorname{Inf}_{P / N}^{P}\left(\varepsilon_{P / N}\left(f_{\mathbf{1}}^{P / N} \operatorname{Def}_{P / N}^{P} a\right)\right) .
$$

Since $\varepsilon_{P}(a)=\sum_{N \unlhd P} f_{N}^{P} \varepsilon_{P}(a)$, the induction hypothesis implies that $f_{N}^{P} \varepsilon_{P}(a)$ lies in $i_{P}\left(\mathbb{F}_{2} R_{\mathbb{Q}}^{*}(P)\right)$, for $N \neq \mathbf{1}$. Thus, one can suppose $a=f_{\mathbf{1}}^{P} a$, i.e. $a \in \partial B^{\times}(P)$. But if $P$ is elementary abelian of rank 2 , or if $P$ has odd order $p^{3}$, then $\partial B^{\times}(P)$ is trivial, by Lemma 6.3 and Corollary 6.10. Hence there is nothing more to prove if $p$ is odd. And for $p=2$, the only case left is when $P$ is dihedral. In that case by Corollary 6.12 , the group $\partial B^{\times}(P)$ has order 2 , generated by the element

$$
v_{P}=\sum_{H \in\left[s_{P}\right]-\{I, J\}} e_{H}^{P}-\left(e_{I}^{P}+e_{J}^{P}\right),
$$

where $\left[s_{P}\right]$ is a set of representatives of conjugacy classes of subgroups of $P$, and where $I$ and $J$ are the elements of $\left[s_{P}\right]$ which have order 2, and are non central in $P$. Checking that $\varepsilon_{P}\left(v_{P}\right)$ lies in $i\left(\mathbb{F}_{2} R_{\mathbb{Q}}^{*}(P)\right)$ is equivalent to checking that $\varepsilon_{P}\left(v_{P}\right)(K(P))=0$ in $\mathbb{F}_{2}$. Since moreover

$$
\left(f_{N}^{P} \varphi\right)(b)=\varphi\left(f_{N}^{P} b\right)
$$

for any $\varphi \in \mathbb{F}_{2} B^{*}(P)$ and $b \in B(P)$, it follows that $\varepsilon_{P}\left(v_{P}\right)\left(f_{N}^{P} K(P)\right)=0$ if $N \neq \mathbf{1}$. In other words, it is enough to check that $\varepsilon_{P}\left(v_{P}\right)(\partial K(P))=0$.

Now $f_{1}^{P} P / H=P / H-P / H Z$, where $Z$ is the centre of $P$. This is 0 if $H \supseteq Z$, so $\partial B(P)$ is the set of elements

$$
\begin{aligned}
\beta & =f_{\mathbf{1}}^{P}(r P / \mathbf{1}+s P / I+t P / J) \\
& =r(P / \mathbf{1}-P / Z)+s(P / I-P / I Z)+t(P / J-P / J Z),
\end{aligned}
$$

for $r, s, t \in \mathbb{Z}$. If such an element $\beta$ is in $K(P)$, then in particular

$$
|\beta|=\frac{|P|}{4}(2 r+s+t)=0,
$$

so $2 r+s+t=0$.

But $\varepsilon_{P}\left(v_{P}\right)(P / H)$ is equal to zero, except if $H$ is conjugate to $I$ or $J$, and then $\varepsilon_{P}\left(v_{P}\right)(P / H)=1$, it follows that $\varepsilon_{P}\left(v_{P}\right)(\beta)=s+t=-2 r$, which is equal to 0 in $\mathbb{F}_{2}$, as was to be shown. This completes the proof. 
Corollary 8.3. The p-biset functor $B^{\times}$is rational.

Proof. Indeed, it is isomorphic to a subfunctor of $\mathbb{F}_{2} R_{\mathbb{Q}}^{*}$, and $\mathbb{F}_{2} R_{\mathbb{Q}}^{*} \cong \operatorname{Hom}_{\mathbb{Z}}\left(R_{\mathbb{Q}}, \mathbb{F}_{2}\right)$. Now $R_{\mathbb{Q}}$ is rational (see Remark 4.8), so its $\mathbb{F}_{2}$-dual and any subfunctor of it are rational (by Remark 4.9), hence $B^{\times}$is rational.

Notation 8.4. Let $\bar{\varepsilon}: B^{\times} \rightarrow \mathbb{F}_{2} R_{\mathbb{Q}}^{*}$ denote the unique morphism such that $i \circ \bar{\varepsilon}=\varepsilon$.

The existence of such a morphism is a consequence of Theorem 8.2. It is injective since $\varepsilon$ is injective.

Theorem 8.5. Let $P$ be a p-group. Then $B^{\times}(P)$ is an elementary abelian 2-group of rank equal to the number of isomorphism classes of rational irreducible representations of $P$ whose type is trivial, cyclic of order 2, or dihedral. More precisely:

(1) If $p \neq 2$, then $B^{\times}(P)=\{ \pm 1\}$.

(2) If $p=2$, then let $g$ be a genetic basis of $P$, and let $\mathscr{H}$ be the subset of $g$ consisting of elements $Q$ such that $N_{P}(Q) / Q$ is trivial, cyclic of order 2 , or dihedral. If $Q \in \mathcal{H}$, then $\partial B^{\times}\left(N_{P}(Q) / Q\right)$ has order 2 , generated by $v_{N_{P}(Q) / Q \text {. Then the }}$ set

$$
\left\{\operatorname{Teninf}_{N_{P}(Q) / Q}^{P} v_{N_{P}(Q) / Q} \mid Q \in \mathscr{H}\right\}
$$

is an $\mathbb{F}_{2}$-basis of $B^{\times}(P)$.

Proof. This follows from the definition of a rational biset functor, and from Corollary 6.12.

8.6. Nilpotent groups. Theorem 8.5 and Lemma 6.3 give the structure (and an explicit $\mathbb{F}_{2}$-basis) of $B^{\times}(G)$ when $G$ is a finite nilpotent group: Theorem 8.5 applies to a Sylow 2-subgroup $G_{2}$ of $G$, and then Lemma 6.3 shows that inflation from $G_{2}$ to $G$ is an isomorphism $B^{\times}\left(G_{2}\right) \rightarrow B^{\times}(G)$.

It might be worth noting that the result can also be expressed using the following natural generalization of the notion of genetic subgroups and genetic basis from $p$ groups to nilpotent groups:

- A finite group $K$ has normal rank 1 if every abelian normal subgroup of $K$ is cyclic.

- Let $G$ be a finite nilpotent group. A Sylow subgroup of $G$ is a Sylow $p$-subgroup of $G$, for some prime $p$. Recall that any subgroup $H$ of $G$ is equal to the direct product of its subgroups $H \cap S$, when $S$ runs through the Sylow subgroups of $G$.

- A subgroup $H$ of $G$ is called genetic if the following two conditions hold:

- The group $N_{G}(H) / H$ has normal rank 1 . 
- If $x \in G$, then $H^{x} \cap Z_{G}(H) \subseteq H$ if and only if $H^{x}=H$, where $Z_{G}(H)$ is the subgroup of $N_{G}(H)$ defined by $Z_{G}(H) / H=Z\left(N_{G}(H) / H\right)$.

With this definition, it is easy to see that $H$ is a genetic subgroup of $G$ if and only if $H \cap S$ is a genetic subgroup of $S$, for each Sylow subgroup $S$ of $G$.

- Two genetic subgroups $H$ and $K$ of $G$ are said to be linked modulo $G$ (notation $H-{ }_{G} K$ ) if there exist $x$ and $y$ in $G$ such that

$$
H^{x} \cap Z_{G}(K) \subseteq K \quad \text { and } \quad K^{y} \cap Z_{G}(H) \subseteq H .
$$

It is easy to see that this happens if and only if $(H \cap S)-_{S}(K \cap S)$ for each Sylow subgroup $S$ of $G$. In particular, the relation $-{ }_{G}$ is an equivalence relation on the set of genetic subgroups of $G$. A genetic basis of $G$ is by definition a set of representatives of equivalence classes for this relation.

If $H$ and $K$ are genetic subgroups of $G$ such that $H-{ }_{G} K$, then the groups $N_{G}(H) / H$ and $N_{G}(K) / K$ are isomorphic. The isomorphism class of this group is called the type of $H$ or $K$, or the type of their equivalence class for $-{ }_{G}$.

- Now the structure theorem for $B^{\times}(G)$ can be stated as follows:

Theorem 8.7. Let $G$ be a finite nilpotent group. Let $\mathrm{g}$ be a genetic basis of $G$, and let $\mathscr{H}$ be the subset of $g$ consisting of elements $H$ such that $N_{G}(H) / H$ is trivial, cyclic of order 2, or a dihedral 2-group. Then the set

$$
\left\{\operatorname{Teninf}_{N_{G}(H) / H}^{G} v_{N_{G}(H) / H} \mid H \in \mathscr{H}\right\}
$$

is an $\mathbb{F}_{2}$-basis of $B^{\times}(G)$.

Proof. Indeed, let $G=G_{\text {odd }} \times S$, where $S$ is a Sylow 2-subgroup of $G$. If $H$ is a subgroup of $G$ such that $N_{G}(H) / H$ is a 2-group, then $H=G_{\text {odd }} \times Q$, for some subgroup $Q$ of $S$. Moreover $N_{G}(H) / H \cong N_{S}(Q) / Q$ in this case, and $H$ is a genetic subgroup of $G$ if and only if $Q$ is a genetic subgroup of $S$. Finally if $Q$ and $R$ are genetic subgroups of $S$, then $\left(G_{\text {odd }} \times Q\right)-_{G}\left(G_{\text {odd }} \times R\right)$ if and only if $Q-_{S} R$.

Remark 8.8. If $G$ is abelian, then it follows easily from the above definitions that there is a unique genetic basis of $G$, consisting of all subgroups $H$ such that $G / H$ is cyclic (see also Example 4.4). So in that case, the rank of $B^{\times}(G)$ is equal to 1 plus the number of subgroups of index 2 in $G$ : this gives a new proof of Matsuda's Theorem (2.11). 


\section{The functorial structure of $B^{\times}$for $p$-groups}

In this section, I will describe the lattice of subfunctors of the $p$-biset functor $B^{\times}$. This result will be a consequence of the description of the lattice of subfunctors of $\mathbb{F}_{2} R_{\mathbb{Q}}$, stated in [7]. It will allow for a complete answer to the question of the surjectivity of the exponential map $B(P) \rightarrow B^{\times}(P)$ for a 2-group $P$ (see 9.7). It will also lead to an interesting connection with the Dade group of $p$-groups, namely the existence of a short exact sequence involving $B^{\times}, \mathbb{F}_{2} R_{\mathbb{Q}}^{*}$, and the $\mathbb{F}_{2}$-reduction $\mathbb{F}_{2} D_{\text {tors }}^{\Omega}$ of the torsion subfunctor of the functor $D^{\Omega}$ of relative syzygies in the Dade group. The reader is referred to [11] for the basic constructions on the Dade group and the functorial approach to it. The $p$-biset functor $D^{\Omega}$ was introduced in [8], where it was shown that it is a quotient of the $\mathbb{Z}$-dual of the Burnside functor.

9.1. Simple functors. Recall first some notation and basic results on simple biset functors: the category of biset functors $\mathcal{F}$ and the category of $p$-biset functors $\mathcal{F}_{p}$ are abelian categories. Their simple objects are parametrized by pairs $(H, V)$, where $H$ is a finite group (in the case of $\mathcal{F}$ ) or a finite $p$-group (in the case of $\mathcal{F}_{p}$ ), and $V$ is a simple $\mathbb{Z} \operatorname{Out}(H)$-module, where $\operatorname{Out}(H)$ is the group of outer automorphisms of $H$ (see e.g. [3], Proposition 2, page 678, or [11], Proposition 7.10 for details). All the simple functors appearing in this section correspond to the case where $V$ is the quotient $\mathbb{Z} / q \mathbb{Z}$ with trivial $\operatorname{Out}(H)$-action, for some prime number $q$. The corresponding simple functor will be denoted by $S_{H, \mathbb{F}_{q}}$.

9.2. The case $\boldsymbol{p} \neq 2$. If $p \neq 2$, there is not much to say, since $B^{\times}(P) \cong \mathbb{F}_{2}$ for any $p$-group $P$, by Assertion 3 of Lemma 6.3. In this case, the functor $B^{\times}$is the constant functor $\Gamma_{\mathbb{F}_{2}}$ introduced in Corollary 8.4 of [11]. It is also isomorphic to the simple functor $S_{1, \mathbb{F}_{2}}$.

In this case moreover, it has been shown by J. Carlson and J. Thévenaz ([13], Theorem 13.3) that for any $p$-group $P$, the torsion part $D_{\text {tors }}(P)$ of the Dade group $D(P)$ is equal to the torsion part $D_{\text {tors }}^{\Omega}(P)$ of the subgroup of $D(P)$ generated by relative syzygies (actually in this case $D(P)=D^{\Omega}(P)$ by Theorem 7.7 of [10]). In this case moreover, by Corollary 8.4 of [11], the functor $\mathbb{F}_{2} R_{\mathbb{Q}}^{*}$ has a unique non zero proper subfunctor, isomorphic to $S_{\mathbf{1}, \mathbb{F}_{2}}$. This yields the following remarkable version of Theorem 11.2 of [11]:

Proposition 9.3. If $p \neq 2$, the injection $\bar{\varepsilon}: B^{\times} \rightarrow \mathbb{F}_{2} R_{\mathbb{Q}}^{*}$ fits into a short exact sequence of p-biset functors

$$
0 \rightarrow B^{\times} \stackrel{\bar{\varepsilon}}{\longrightarrow} \mathbb{F}_{2} R_{\mathbb{Q}}^{*} \rightarrow D_{\text {tors }} \rightarrow 0,
$$

where $D_{\text {tors }}$ is the torsion part of the Dade p-biset functor. 
9.4. The case $p=2$. There is a bilinear pairing

$$
\langle,\rangle: \mathbb{F}_{2} R_{\mathbb{Q}}^{*} \times \mathbb{F}_{2} R_{\mathbb{Q}} \rightarrow \mathbb{F}_{2} .
$$

This means that for each 2-group $P$, there is a bilinear form

$$
\langle,\rangle_{P}: \mathbb{F}_{2} R_{\mathbb{Q}}^{*}(P) \times \mathbb{F}_{2} R_{\mathbb{Q}}(P) \rightarrow \mathbb{F}_{2},
$$

with the property that for any 2-group $Q$, for any $f \in \operatorname{Hom}_{\mathcal{C}_{p}}(P, Q)$, for any $a \in$ $\mathbb{F}_{2} R_{\mathbb{Q}}^{*}(P)$ and any $b \in \mathbb{F}_{2} R_{\mathbb{Q}}(Q)$, one has that

$$
\left\langle\mathbb{F}_{2} R_{\mathbb{Q}}^{*}(f)(a), b\right\rangle_{Q}=\left\langle a, \mathbb{F}_{2} R_{\mathbb{Q}}\left(f^{\mathrm{op}}\right)(b)\right\rangle_{P} .
$$

Moreover this pairing is non-degenerate: this means that for any 2-group $P$, the pairing $\langle,\rangle_{P}$ is non-degenerate. In particular, each subfunctor $F$ of $\mathbb{F}_{2} R_{\mathbb{Q}}^{*}$ is isomorphic to $\mathbb{F}_{2} R_{\mathbb{Q}} / F^{\perp}$, where $F^{\perp}$ is the orthogonal of $F$ for the pairing $\langle$,$\rangle .$

In particular, the lattice of subfunctors of $\mathbb{F}_{2} R_{\mathbb{Q}}^{*}$ is isomorphic to the opposite lattice of the lattice of subfunctors of $\mathbb{F}_{2} R_{\mathbb{Q}}$. Now since $B^{\times}$is isomorphic to a subfunctor of $\mathbb{F}_{2} R_{\mathbb{Q}}$, its lattice of subfunctors is isomorphic to the opposite lattice of the lattice of subfunctors of $\mathbb{F}_{2} R_{\mathbb{Q}}$ containing $B^{\sharp}=\left(B^{\times}\right)^{\perp}$. By Theorem 4.4 of [7], any subfunctor $L$ of $\mathbb{F}_{2} R_{\mathbb{Q}}$ is equal to the sum of all subfunctors $H_{Q}$ it contains, where $Q$ is a 2-group of normal 2-rank 1 , and $H_{Q}$ is the subfunctor of $\mathbb{F}_{2} R_{\mathbb{Q}}$ generated by the image $\bar{\Phi}_{Q}$ in $\mathbb{F}_{2} R_{\mathbb{Q}}(Q)$ of the unique (up to isomorphism) irreducible rational faithful $\mathbb{Q} Q$-module $\Phi_{Q}$.

In particular $B^{\sharp}$ is the sum of the subfunctors $H_{Q}$, where $Q$ is a 2-group of normal 2-rank 1 such that $\bar{\Phi}_{Q} \in B^{\sharp}(Q)$. This means that $\left\langle a, \bar{\Phi}_{Q}\right\rangle_{Q}=0$, for any $a \in B^{\times}(Q)$. Now $\Phi_{Q}=f_{1} \Phi_{Q}$ since $\Phi_{Q}$ is faithful, so

$$
\left\langle a, \bar{\Phi}_{Q}\right\rangle_{Q}=\left\langle a, f_{\mathbf{1}}^{Q} \bar{\Phi}_{Q}\right\rangle_{Q}=\left\langle f_{\mathbf{1}}^{Q} a, \bar{\Phi}_{Q}\right\rangle_{Q},
$$

because $f_{\mathbf{1}}^{Q}=\left(f_{\mathbf{1}}^{Q}\right)^{\text {op }}$. Thus $\bar{\Phi}_{Q} \in B^{\sharp}(Q)$ if and only if $\bar{\Phi}_{Q}$ is orthogonal to $\partial B^{\times}(Q)$. Since $Q$ has normal 2-rank 1, this is always the case by Corollary 6.12, except possibly if $Q$ is trivial, cyclic of order 2, or dihedral (of order at least 16). Now $H_{1}=H_{C_{2}}=\mathbb{F}_{2} R_{\mathbb{Q}}$ by Theorem 5.6 of [7]. Since $B^{\times}$is not the zero subfunctor of $\mathbb{F}_{2} R_{\mathbb{Q}}$, it follows that $H_{Q} \nsubseteq B^{\sharp}$, if $Q$ is trivial or cyclic of order 2. Now if $Q$ is dihedral, then $\Phi_{Q}$ is equal to $\mathbb{Q} Q / I-\mathbb{Q} Q / I Z$, where $I$ is a non-central subgroup of order 2 of $Q$, and $Z$ is the centre of $Q$ (see Proposition 3.7 of [7]). Now

$$
\left\langle\bar{\varepsilon}_{Q}\left(v_{Q}\right), \bar{\Phi}_{Q}\right\rangle=\varepsilon_{Q}\left(v_{Q}\right)(Q / I-Q / I Z)=1-0=1,
$$

It follows that $H_{Q} \nsubseteq B^{\sharp}$ if $Q$ is dihedral. Finally $B^{\sharp}$ is the sum of all subfunctors $H_{Q}$, where $Q$ is cyclic of order at least 4 , or generalized quaternion, or semi-dihedral. 
Recall from Theorem 6.2 of [7] that the poset of proper subfunctors of $\mathbb{F}_{2} R_{\mathbb{Q}}$ is isomorphic to the poset of closed subsets of the following graph:

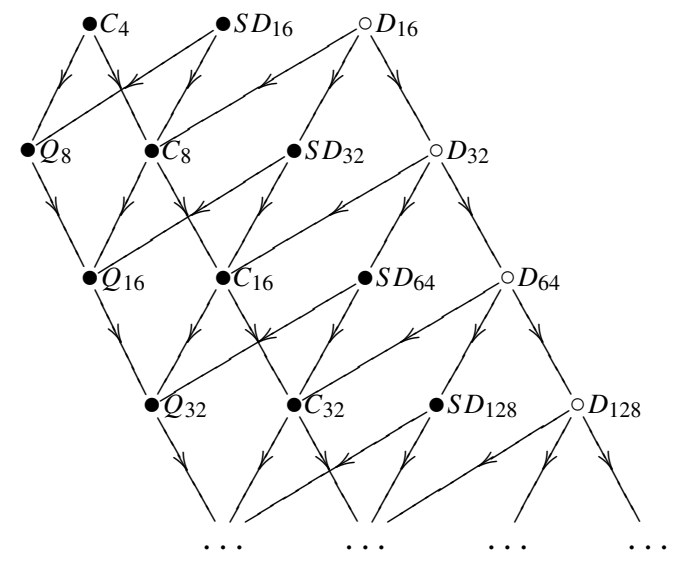

The vertices of this graph are the isomorphism classes of groups of normal 2-rank 1 and order at least 4, and there is an arrow from vertex $Q$ to vertex $R$ if and only if $H_{R} \subseteq H_{Q}$. The vertices with a filled $\bullet$ are exactly labelled by the groups $Q$ for which $H_{Q} \subseteq B^{\sharp}$, and the vertices with a $\circ$ are labelled by dihedral groups.

By the above remarks, the lattice of subobjects of $B^{\times}$is isomorphic to the opposite lattice of the lattice of subfunctors of $\mathbb{F}_{2} R_{\mathbb{Q}}$ containing $B^{\sharp}$. Thus:

Theorem 9.5. The p-biset functor $B^{\times}$is uniserial. It has an infinite strictly increasing series of proper subfunctors

$$
0 \subset L_{0} \subset L_{1} \cdots \subset L_{n} \subset \cdots
$$

where $L_{0}$ is generated by the element $v_{1}$, and $L_{i}$, for $i>0$, is generated by the element $v_{D_{2^{i+3}}}$ of $B^{\times}\left(D_{2^{i+3}}\right)$. The functor $L_{0}$ is isomorphic to the simple functor $S_{\mathbf{1}, \mathbb{F}_{2}}$, and the quotient $L_{i} / L_{i-1}$, for $i \geq 1$, is isomorphic to the simple functor $S_{D_{2^{i+3}}, \mathbb{F}_{2}}$.

Proof. Indeed $L_{0}^{\perp}=B^{\sharp}+H_{D_{16}}$ is the unique maximal proper subfunctor of $\mathbb{F}_{2} R_{\mathbb{Q}}$. Thus $L_{0}$ is isomorphic to the unique simple quotient of $\mathbb{F}_{2} R_{\mathbb{Q}}$, which is $S_{\mathbf{1}, \mathbb{F}_{2}}$ by Proposition 5.1 of [7]. Similarly for $i \geq 1$, the simple quotient $L_{i} / L_{i-1}$ is isomorphic to the quotient

$$
\left.\left(B^{\sharp}+H_{D_{2^{i+3}}}\right) / B^{\sharp}+H_{D_{2^{i+4}}}\right),
$$

which is a quotient of

$$
\left(B^{\sharp}+H_{D_{2^{i+3}}}\right) / B^{\sharp} \cong H_{D_{2^{i+3}}} /\left(B^{\sharp} \cap H_{D_{2^{i+3}}}\right) .
$$

But the only simple quotient of $H_{D_{2^{i+3}}}$ is $S_{D_{2^{i+3}}, \mathbb{F}_{2}}$, by Proposition 5.1 of [7] again. 
Remark 9.6. Let $P$ be a 2-group. By Theorem 5.12 of [7], the $\mathbb{F}_{2}$-dimension of $S_{\mathbf{1}, \mathbb{F}_{2}}(P)$ is equal to the number of isomorphism classes of rational irreducible representations of $P$ whose type is 1 or $C_{2}$, whereas the $\mathbb{F}_{2}$-dimension of $S_{D_{2 i+3}, \mathbb{F}_{2}}(P)$ is the number of isomorphism classes of rational irreducible representations of $P$ whose type is isomorphic to $D_{2^{i+3}}$. This gives a way to recover Theorem 8.5: the $\mathbb{F}_{2}$-dimension of $B^{\times}(P)$ is equal to the number of isomorphism classes of rational irreducible representations of $P$ whose type is trivial, cyclic of order 2 , or dihedral.

9.7. The surjectivity of the exponential map. Let $G$ be a finite group. The exponential map $\exp _{G}: B(G) \rightarrow B^{\times}(G)$ is defined in Section 7 of Yalçın's paper ([24]) by

$$
\exp _{G}(x)=(-1) \uparrow x,
$$

where $-1=-\mathbf{1} / \mathbf{1} \in B^{\times}(\mathbf{1})$, and where the exponentiation

$$
(y, x) \in B^{\times}(G) \times B(G) \mapsto y \uparrow x \in B^{\times}(G)
$$

is defined by extending the usual exponential map $(Y, X) \mapsto Y^{X}$, where $X$ and $Y$ are $G$-sets, and $Y^{X}$ is the set of maps from $X$ to $Y$, with $G$-action given by $(g \cdot f)(x)=g f\left(g^{-1} x\right)$.

Yalçın ([24], Theorem 7.6) shows that if the 2-group $P$ has no subquotient isomorphic to the dihedral group $D_{2^{n}}$, for $n \geq 4$, then the map $\exp _{P}$ is surjective, but he also gives an example ([24], Lemma 7.9) of a 2-group admitting a subquotient isomorphic to $D_{16}$ for which the map $\exp _{P}$ is surjective.

It is possible to refine Yalçın's result, by giving a necessary and sufficient condition for the surjectivity of the map $\exp _{P}$ for a 2-group $P$. The first step in doing this is the following alternative description of the map $\exp _{\mathrm{G}}$, for an arbitrary finite group $G$ : the group $B(G)$ is naturally isomorphic to $\operatorname{Hom}_{\mathcal{C}}(\mathbf{1}, G)$, by considering any $G$-set as a $(G, \mathbf{1})$-biset. It is clear that if $X$ is a finite $G$-set, and $Y$ is a finite set, then

$$
T_{X}(Y)=Y^{X},
$$

where $T_{X}(Y)=\operatorname{Hom}_{1}\left(X^{\mathrm{op}}, Y\right)$ as in Section 5 .

Equality 9.8 can be extended by linearity, to show that for any $x \in B(G)$

$$
\exp _{G}(x)=(-1) \uparrow x=B^{\times}(x)(-1) .
$$

In particular the image $\operatorname{Im}\left(\exp _{G}\right)$ of the exponential map $\exp _{G}$ is equal to $\operatorname{Hom}_{\mathcal{C}}(1, G)(-1)$. Denoting by $J$ the sub-biset functor of $B^{\times}$generated by $-1 \in$ $B^{\times}(\mathbf{1})$ (see 3.11), it is now clear that $\operatorname{Im}\left(\exp _{G}\right)=J(G)$ for any finite group $G$.

Now the restriction of the functor $J$ to the category $\mathcal{C}_{2}$ is equal to $L_{0}$, which is isomorphic to the simple functor $S_{1, \mathbb{F}_{2}}$. By Theorem 5.12 and Remark 5.13 of [7], if $P$ is a finite 2-group, then the $\mathbb{F}_{2}$-dimension of $S_{1, \mathbb{F}_{2}}(P)$ is equal to the number of 
isomorphism classes whose type is trivial or cyclic of order 2, and this is also equal to the number of isomorphism classes of absolutely irreducible rational representations of $P$. This gives finally:

Proposition 9.9. Let $P$ be a finite 2-group. Then:

(1) The $\mathbb{F}_{2}$-dimension of the image of the exponential map

$$
\exp _{P}: B(P) \rightarrow B^{\times}(P)
$$

is equal to the number of isomorphism classes of absolutely irreducible rational representations of $P$.

(2) The map $\exp _{P}$ is surjective if and only if the group $P$ has no irreducible rational representation of dihedral type, or equivalently, no genetic subgroup $Q$ such that $N_{P}(Q) / Q$ is dihedral.

9.10. Connection with the Dade group. Proposition 9.3 stated an interesting connection between the $p$-biset functors $B^{\times}, \mathbb{F}_{2} R_{\mathbb{Q}}^{*}$ and $D_{\text {tors }}$, when $p$ is an odd prime. Theorem 9.5 is an extension of this result to the case $p=2$. So in this section $p$ denotes an arbitrary prime number.

First recall the following notation: if $P$ is a finite $p$-group, and $X$ is a finite $P$-set, denote by $\omega_{X}$ the element of $B^{*}(P)$ defined by $\omega_{X}(P / Q)=1$ if $X^{Q} \neq 0$, and $\omega_{X}(P / Q)=0$ otherwise. Denote by $\Omega_{X}$ the class in the Dade group $D(P)$ of the syzygy relative to $X$ of the trivial module, and by $D^{\Omega}(P)$ the subgroup of $D(P)$ generated by these relative syzygies. Recall from [8] (Theorem 1.7 and Theorem 1.8) that $D^{\Omega}(P)$ is the evaluation at $P$ of a $p$-biset functor, and that there exists a surjective morphism of $p$-biset functors $\Theta$ from $B^{*}$ to $D^{\Omega}$ such that $\Theta_{P}\left(\omega_{X}\right)=\Omega_{X}$, for any finite $p$-group $P$ and finite $P$-set $X$. This yields an exact sequence of $p$-biset functors

$$
0 \rightarrow R_{\mathbb{Q}}^{*} \rightarrow B^{*} \rightarrow D^{\Omega} / D_{\text {tors }}^{\Omega} \rightarrow 0,
$$

where $D_{\text {tors }}^{\Omega}$ is the torsion subfunctor of $D^{\Omega}$. This shows that $\theta=\Theta \circ i$ is a surjective morphism of $p$-biset functors $R_{\mathbb{Q}}^{*} \rightarrow D_{\text {tors }}^{\Omega}$. Denote by $\bar{\theta}: \mathbb{F}_{2} \mathbb{R}_{\mathbb{Q}}^{*} \rightarrow \mathbb{F}_{2} D^{\Omega}$ the $\mathbb{F}_{2}$-reduction of this map.

Proposition 9.11. Let $p$ be a prime number. There is an exact sequence of p-biset functors:

$$
0 \rightarrow B^{\times} \stackrel{\bar{\varepsilon}}{\longrightarrow} \mathbb{F}_{2} R_{\mathbb{Q}}^{*} \stackrel{\bar{\theta}}{\longrightarrow} \mathbb{F}_{2} D_{\text {tors }}^{\Omega} \rightarrow 0 .
$$

Proof. In the case $p \neq 2$, this proposition is equivalent to Proposition 9.3, because $\mathbb{F}_{2} D_{\text {tors }}^{\Omega}=\mathbb{F}_{2} D_{\text {tors }} \cong D_{\text {tors }}$ in this case.

To prove the proposition in the case $p=2$, it is enough to show that the image of $\bar{\varepsilon}$ is contained in the kernel of $\bar{\theta}$, and that for any 2-group $P$, the $\mathbb{F}_{2}$-dimension of 
$\mathbb{F}_{2} R_{\mathbb{Q}}^{*}(P)$ is equal to the sum of the $\mathbb{F}_{2}$-dimensions of $B^{\times}(P)$ and $\mathbb{F}_{2} D_{\text {tors }}^{\Omega}(P)$ : but by Corollary 7.6 of [9], there is a group isomorphism

$$
D_{\text {tors }}^{\Omega}(P) \cong(\mathbb{Z} / 4 \mathbb{Z})^{a_{P}} \oplus(\mathbb{Z} / 2 \mathbb{Z})^{b_{P}},
$$

where $a_{P}$ is equal to the number of isomorphism classes of rational irreducible representations of $P$ whose type is generalized quaternion, and $b_{P}$ equal to the number of isomorphism classes of rational irreducible representations of $P$ whose type is cyclic of order at least 3 , or semi-dihedral. Thus

$$
\operatorname{dim}_{\mathbb{F}_{2}} \mathbb{F}_{2} D_{\text {tors }}^{\Omega}(P)=a_{P}+b_{P} .
$$

Now since $\operatorname{dim}_{\mathbb{F}_{2}} B^{\times}(P)$ is equal to the number of isomorphism classes of rational irreducible representations of $P$ whose type is cyclic of order at most 2, or dihedral, it follows that $\operatorname{dim}_{\mathbb{F}_{2}} \mathbb{F}_{2} D_{\text {tors }}^{\Omega}(P)+\operatorname{dim}_{\mathbb{F}_{2}} B^{\times}(P)$ is equal to the number of isomorphism classes of rational irreducible representations of $P$, i.e. to $\operatorname{dim}_{\mathbb{F}_{2}} \mathbb{F}_{2} R_{\mathbb{Q}}^{*}(P)$.

So the only thing to check to complete the proof, is that the image of $\bar{\varepsilon}$ is contained in the kernel of $\bar{\theta}$. Since $B^{\times}, \mathbb{F}_{2} R_{\mathbb{Q}}^{*}$ and $\mathbb{F}_{2} D_{\text {tors }}^{\Omega}$ are rational 2-biset functors, it suffices to check that if $P$ is a 2-group of normal 2-rank 1, and $a \in \partial B^{\times}(P)$, then the image

of $a$ in $\partial \mathbb{F}_{2} R_{\mathbb{Q}}^{*}(P)$ lies in the kernel of $\bar{\theta}$. There is nothing to do if $P$ is generalized quaternion, or semi-dihedral, or cyclic of order at least 3 , for in this case $\partial B^{\times}(P)=0$ by Corollary 6.10. Now if $P$ is cyclic of order at most 2 , then $D^{\Omega}(P)=\{0\}$, and the result follows. And if $P$ is dihedral, then $D^{\Omega}(P)$ is torsion free by Theorem 10.3 of [12], so $D_{\text {tors }}^{\Omega}(P)=\{0\}$ again.

Acknowledgements. I wish to thank the referee for his careful reading of this paper, and his detailed report. I also thank Jacques Thévenaz for stimulating discussions and many useful suggestions, and the Bernoulli Center at EPFL, where the first version of this paper was completed in April 2005.

\section{References}

[1] J. Beidleman, H. Heineken, and M. Newell, Centre and norm. Bull. Austral. Math. Soc. 69 (3) (2004), 457-464. Zbl 1060.20020 MR 2066664

[2] S. Bouc, Exponentielle et modules de Steinberg. J. Algebra 150 (1) (1992), 118-157. Zbl 0759.19001 MR 1174892

[3] S. Bouc, Foncteurs d'ensembles munis d'une double action. J. Algebra 183 (0238) (), 664-736, 1996. Zbl 0858.19001 MR 1401673

[4] S. Bouc, Burnside rings. In Handbook of Algebra, Volume 2, Chapter 6D, North-Holland, Amsterdam 2000 2000, 739-804. Zbl 0969.19001 MR 1759611

[5] S. Bouc, Non-additive exact functors and tensor induction for Mackey functors. Mem. Amer. Math. Soc. 144, no. 683, (2000). Zbl 0949.19001 MR 1662073 
[6] S. Bouc, A remark on a theorem of Ritter and Segal. J. Group Theory 4 (2001), 11-18. Zbl 0976.20001 MR 1808835

[7] S. Bouc, The functor of rational representations for $p$-groups. Adv. in Math. 186 (2004), 267-306. Zbl 1055.20005 MR 2073907

[8] S. Bouc, A remark on the Dade group and the Burnside group. J. Algebra 279 (1) (2004), 180-190. Zbl 1059.19001 MR 2078393

[9] S. Bouc, Biset functors and genetic sections for p-groups. J. Algebra 284 (1) (2005), 179-202. Zbl 1062.19001 MR 2115011

[10] S. Bouc, The Dade group of a p-group. Invent. Math. 164 (2006), 189-231.Zbl 1099.20004 MR 2207787

[11] S. Bouc and J. Thévenaz, The group of endo-permutation modules. Invent. Math. 139 (2000), 275-349. Zbl 0954.20002 MR 1738450

[12] J. Carlson and J. Thévenaz, Torsion endo-trivial modules. Algebr. Represent. Theory 3 (2000), 303-335. Zbl 0970.20004 MR 1808129

[13] J. Carlson and J. Thévenaz, The classification of torsion endo-trivial modules. Ann. of Math. 162 (2) (2005), 823-883. Zbl 05042687 MR 2183283

[14] R. Dedekind, Über Gruppen, deren sämmtliche Theiler Normaltheiler sind. Math. Ann. 48 (4) (1897), 548-561. JFM 28.0129.03 MR 1510943

[15] A. Dress, A characterization of solvable groups. Math. Z. 110 (1969), 213-217. Zbl 0174.30806 MR 0248239

[16] D. Gluck, Idempotent formula for the Burnside ring with applications to the $p$-subgroup simplicial complex. Illinois J. Math. 25 (1981), 63-67. Zbl 0424.16007 MR 0602896

[17] W. Gorenstein, Finite groups. Harper \& Row, Publishers, New York, London 1968. Zbl 0185.05701 MR 0231903

[18] T. Matsuda, On the unit group of Burnside rings. Japan. J. Math. 8 (1) (1982), 71-93. Zbl 0517.16008 MR 0722522

[19] T. Matsuda and T. Miyata, On the unit groups of the Burnside rings of finite groups. $J$. Math. Soc. Japan 35 (1) (1983), 345-354. Zbl 0491.20004 MR 0692332

[20] J. Ritter, Ein Induktionssatz für rational Charaktere von nilpotenten Gruppen. J. Reine Angew. Math. 254 (1972), 133-151. Zbl 0242.20003 MR 0470058

[21] G. Segal, Permutation representations of finite p-groups. Quart. J. Math. Oxford 23 (1972), 375-381. Zbl 0338.20017 MR 0322041

[22] J. Thévenaz, Permutation representation arising from simplicial complexes. J. Combin. Theory 46 (1987), 122-155. Ser.A. Zbl 0638.20006 MR 0899904

[23] T. tom Dieck, Transformation groups and representation theory, Lecture Notes in Math. 766, Springer-Verlag, Berlin 1979. Zbl 0445.57023 MR 0551743

[24] E. Yalçın, An induction theorem for the unit groups of Burnside rings of 2-groups. $J$. Algebra 289 (2005), 105-127. Zbl 1076.19002 MR 2139093

[25] T. Yoshida, Idempotents in Burnside rings and Dress induction theorem. J. Algebra 80 (1983), 90-105. Zbl 0521.20003 MR 0690705 
Vol. $82(2007)$

[26] T. Yoshida, On the unit groups of Burnside rings. J. Math. Soc. Japan 42 (1) (1990), 31-64. Zbl 0694.20009 MR 1027539

Received April 19, 2005

Serge Bouc, CNRS-LAMFA, Université de Picardie-Jules Verne 33, rue St Leu, 80039 Amiens Cedex 1, France

E-mail: serge.bouc@u-picardie.fr 